\title{
Investigating the physical properties of transiting hot Jupiters with the 1.5-m Kuiper Telescope
}

\author{
Jake D. Turner, ${ }^{1,2 \star}$ Robin M. Leiter, ${ }^{1}$ Lauren I. Biddle, ${ }^{2,3}$ Kyle A. Pearson, ${ }^{4}$ \\ Kevin K. Hardegree-Ullman, ${ }^{5}$ Robert M. Thompson, ${ }^{2}$ Johanna K. Teske,${ }^{2} \dagger$ \\ Ian T. Cates, ${ }^{2}$ Kendall L. Cook, ${ }^{2}$ Michael P. Berube, ${ }^{2}$ Megan N. Nieberding, ${ }^{2}$ \\ Christen K. Jones, ${ }^{2}$ Brandon Raphael, ${ }^{2}$ Spencer Wallace, ${ }^{2}$ Zachary T. Watson ${ }^{2}$ \\ and Robert E. Johnson ${ }^{6}$ \\ ${ }^{1}$ Department of Astronomy, University of Virginia, Charlottesville, VA 22904, USA \\ ${ }^{2}$ Steward Observatory, University of Arizona, Tucson, AZ 85721, USA \\ ${ }^{3}$ Department of Physics and Astronomy, Northern Arizona University, Flagstaff, AZ 86011, USA \\ ${ }^{4}$ Lunar and Planetary Laboratory, University of Arizona, Tucson, AZ 85721, USA \\ ${ }^{5}$ Department of Physics and Astronomy, University of Toledo, Toledo, $\mathrm{OH} 43606$, USA \\ ${ }^{6}$ Department of Materials Science and Engineering, University of Virginia, Charlottesville, VA 22904, USA
}

Accepted 2017 August 25. Received 2017 August 24; in original form 2016 December 27

\begin{abstract}
We present new photometric data of 11 hot Jupiter transiting exoplanets (CoRoT-12b, HATP-5b, HAT-P-12b, HAT-P-33b, HAT-P-37b, WASP-2b, WASP-24b, WASP-60b, WASP-80b, WASP-103b and XO-3b) in order to update their planetary parameters and to constrain information about their atmospheres. These observations of CoRoT-12b, HAT-P-37b and WASP$60 \mathrm{~b}$ are the first follow-up data since their discovery. Additionally, the first near-UV transits of WASP-80b and WASP-103b are presented. We compare the results of our analysis with previous work to search for transit timing variations (TTVs) and a wavelength dependence in the transit depth. TTVs may be evidence of a third body in the system, and variations in planetary radius with wavelength can help constrain the properties of the exoplanet's atmosphere. For WASP-103b and XO-3b, we find a possible variation in the transit depths which may be evidence of scattering in their atmospheres. The $B$-band transit depth of HAT-P-37b is found to be smaller than its near-IR transit depth and such a variation may indicate TiO/VO absorption. These variations are detected from $2-4.6 \sigma$, so follow-up observations are needed to confirm these results. Additionally, a flat spectrum across optical wavelengths is found for five of the planets (HAT-P-5b, HAT-P-12b, WASP-2b, WASP-24b and WASP-80b), suggestive that clouds may be present in their atmospheres. We calculate a refined orbital period and ephemeris for all the targets, which will help with future observations. No TTVs are seen in our analysis with the exception of WASP-80b and follow-up observations are needed to confirm this possible detection.
\end{abstract}

Key words: techniques: photometric - planets and satellites: atmospheres - planets and satellites: gaseous planets - planet-star interactions.

\section{INTRODUCTION}

To date, over 3400 exoplanets have been discovered (NASA Exoplanet Archive; Akeson et al. 2013) and most of these planets have been found using the transit method (e.g. Charbonneau

*E-mail: jt6an@virginia.edu

$\dagger$ Origins Fellow at Carnegie DTM/OCIW et al. 2000; Henry et al. 2000) in large-scale transit surveys such as Kepler (Borucki et al. 2010), K2 (Howell et al. 2014), WASP (Pollacco et al. 2006; Collier Cameron et al. 2007) and CoRoT (Baglin 2003; Moutou et al. 2013). Transiting exoplanet systems (TEPs) are of great interest because their radius can be directly measured in relation to their star with photometric observations (Charbonneau et al. 2000; Henry et al. 2000). With the addition of spectroscopic and radial velocity measurements, many physical properties of TEP systems (mass, radius, semimajor axis, gravity, 
Table 1. Journal of observations.

\begin{tabular}{|c|c|c|c|c|c|c|c|c|c|}
\hline $\begin{array}{l}\text { Planet } \\
\text { name }\end{array}$ & $\begin{array}{l}\text { Date } \\
\text { (UT) }\end{array}$ & Filter $^{1}$ & $\begin{array}{l}\text { Cadence } \\
\text { (s) }\end{array}$ & $N_{\mathrm{pts}}$ & $\begin{array}{c}\text { OoT RMS } \\
\text { (mmag) }\end{array}$ & $\begin{array}{c}\text { Res RMS }^{3} \\
(\mathrm{mmag})\end{array}$ & $\begin{array}{l}\text { Seeing } \\
(\operatorname{arcsec})\end{array}$ & $k^{a}$ & $\chi_{r}^{2 b}$ \\
\hline CoRoT-12b & 2013 February 15 & $R$ & 60.39 & 265 & 6.06 & 4.99 & $1.96-4.06$ & 4 & 1.32 \\
\hline HAT-P-5b & 2015 June 6 & $U$ & 80.64 & 191 & 3.02 & 2.84 & $1.36-2.17$ & 4 & 0.97 \\
\hline HAT-P-12b & 2014 January 19 & $B$ & 188.25 & 89 & 1.13 & 1.27 & $2.22-2.96$ & 7 & 1.99 \\
\hline HAT-P-33b & 2012 April 6 & $R$ & 24.29 & 706 & 3.65 & 4.15 & $1.01-2.68$ & 4 & 7.84 \\
\hline HAT-P-37b & 2015 July 1 & $B$ & 115.51 & 128 & 2.37 & 2.88 & $0.98-2.45$ & 4 & 1.10 \\
\hline HAT-P-37b & 2015 July 1 & $R$ & 115.51 & 129 & 2.33 & 2.30 & $0.98-2.45$ & 4 & 1.33 \\
\hline WASP-2b & 2014 June 14 & $B$ & 59.31 & 230 & 2.41 & 2.28 & $1.43-2.68$ & 7 & 3.55 \\
\hline WASP-24b & 2012 March 23 & $R$ & 24.95 & 531 & 2.41 & 2.55 & $1.03-2.12$ & 7 & 4.06 \\
\hline WASP-24b & 2012 April 6 & $R$ & 26.77 & 434 & 5.14 & 5.73 & $1.01-2.72$ & 6 & 2.04 \\
\hline WASP-60b & 2012 December 1 & $B$ & 20.46 & 751 & 5.30 & 4.65 & $0.83-6.82$ & 4 & 1.21 \\
\hline WASP-80b & 2014 June 16 & $U$ & 93.50 & 160 & 7.76 & 7.20 & $1.54-2.92$ & 7 & 1.15 \\
\hline WASP-103b & 2015 June 3 & $U$ & 65.05 & 363 & 3.66 & 3.62 & $1.37-2.56$ & 6 & 1.29 \\
\hline $\mathrm{XO}-3 \mathrm{~b}$ & 2012 November 30 & $B$ & 40.63 & 418 & 2.04 & 2.20 & $1.44-2.23$ & 4 & 1.96 \\
\hline
\end{tabular}

${ }^{1}$ Filter: $B$ is the Harris $B(330-550 \mathrm{~nm}), R$ is the Harris $R(550-900 \mathrm{~nm})$ and $U$ is the Bessell $U(303-417 \mathrm{~nm}) .{ }^{2}$ OoT RMS relative flux.

${ }^{3}$ Residual (res) RMS flux after subtracting the EXOMOP best-fitting model from the data.

${ }^{a} k$ is the degrees of freedom used in the EXOMOP best-fitting model.

${ }^{b}$ Reduced $\chi^{2}\left(\chi_{r}^{2}\right)$ calculated using the EXOMOP best-fitting model, $N_{\mathrm{pts}}$, and $k$.

temperature, eccentricity, orbital period) can be directly measured (e.g. Charbonneau et al. 2007). Additionally, multiple-band photometry of a TEP system can be used to constrain the composition of an exoplanet's atmosphere (Seager \& Sasselov 2000; Brown 2001; Hubbard et al. 2001; Charbonneau et al. 2002). The absorption properties of different species in a planetary atmosphere vary with wavelength, causing an observable variation in the planet's radius. Photometric light-curve analysis can also be used to search for transit timing variations (TTVs). TTVs can indicate additional bodies in a TEP system or an unstable orbit caused by tidal forces from the star (e.g. Miralda-Escudé 2002; Holman \& Murray 2005; Holman et al. 2010).

In this work, we present new ground-based photometric data of 11 confirmed transiting hot Jupiter exoplanets. We describe and perform TEP modelling techniques (Sections 2 and 3) to determine the orbital and physical parameters of each system, and compare our results with previous published results to confirm and improve the planetary parameters (Sections 4 and 5). For each system, we combine our results with previous work to search for a variation in planetary radius with wavelength (Section 6), which could indicate Rayleigh scattering, the presence of an absorptive atmosphere, or clouds. Finally, we combine our mid-transit data with previous observations to recalculate each system's orbital period and search for TTVs.

\section{OBSERVATIONS AND DATA REDUCTION}

All the observations were performed at the University of Arizona's Steward Observatory 1.55-m Kuiper Telescope on Mt Bigelow near Tucson, Arizona. The Mont $4 \mathrm{k}$ CCD has a field of view of 9.7 arcmin $\times 9.7$ arcmin and contains a $4096 \times 4096$ pixel sensor. The CCD is binned $3 \times 3$ to achieve a resolution of 0.43 arcsec pixel ${ }^{-1}$ and binning reduces the read-out time to $\sim 10 \mathrm{~s}$. Our observations were taken with the Bessell $U(303-417 \mathrm{~nm})$, Harris $B(360-500 \mathrm{~nm})$ and Harris $R(550-900 \mathrm{~nm})$ photometric band filters. To ensure accurate timing in these observations, the clocks were synchronized with a GPS every few seconds. In all the data sets, the average shift in the centroid of our targets is less than 0.6 pixels $(0.26$ arcsec) due to excellent autoguiding (the maximum is 3.4 pixels). This telescope has been used extensively in exo- planet transit studies (Dittmann et al. 2009a,b, 2010, 2012; Scuderi et al. 2010; Teske et al. 2013; Turner et al. 2013, 2016b; Biddle et al. 2014; Pearson, Turner \& Sagan 2014; Zellem et al. 2015). A summary of all our observations are displayed in Table 1.

To reduce the data and create the light curves, we use the reduction pipeline ExoDRPL ${ }^{1}$ (Pearson et al. 2014). Each of our images are bias-subtracted and flat-fielded with 10 biases and flats. To produce the light curve for each observation, we perform aperture photometry (using phot in the IRAF ${ }^{2}$ DAOPHOT package) by measuring the flux from our target star as well as the flux from eight different reference stars with 110 different circular aperture radii. The aperture radii sizes we explore are different for every observation due to changes in seeing conditions. For the analysis, a constant sky annulus for every night of observation of each target is chosen (a different sky annulus is used depending on the seeing and the crowdedness of the target field) to measure the brightness of the sky during the observations. We reduce the risk of contamination by making sure no stray light from the target star or other nearby stars falls in the chosen aperture. A synthetic reference light curve is produced by averaging the light curves from our reference stars. The final light curve of each date is normalized by dividing by this synthetic light curve to correct for any systematic differences from atmospheric variations (i.e. airmass) throughout the night. Every combination of reference stars and aperture radii are considered and we systematically choose the best aperture and reference stars by minimizing the scatter in the out-of-transit (OoT) data points. The $1 \sigma$ error bars on the data points include the readout noise, flat-fielding errors and Poisson noise. The final light curves are presented in Figs 13. The data points of all our transits are available in electronic form (see Table 2). For all the transits, the OoT baselines have a photometric root-mean-squared (RMS) value between 1.13 and $7.76 \mathrm{mmag}$.

\footnotetext{
${ }^{1}$ https://sites.google.com/a/email.arizona.edu/kyle-pearson/exodrpl

2 IRAF is distributed by the National Optical Astronomy Observatory, which is operated by the Association of Universities for Research in Astronomy, Inc., under cooperative agreement with the National Science Foundation.
} 

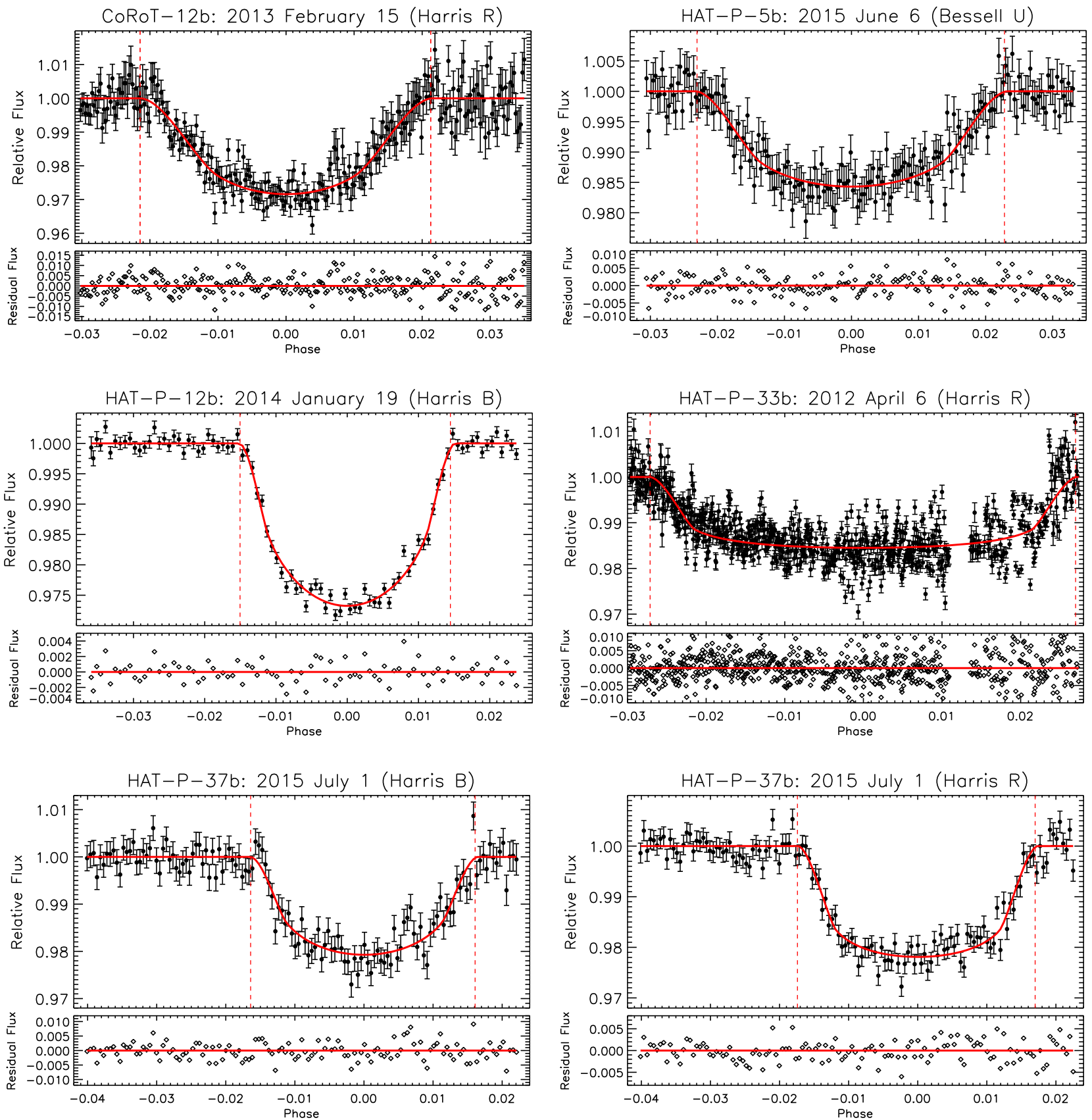

Figure 1. Light curves of CoRoT-12b, HAT-P-5b, HAT-P-12b, HAT-P-33b and HAT-P-37b. The $1 \sigma$ error bars include the readout noise, the Poisson noise and the flat-fielding error. The best-fitting models obtained from the EXOplanet MOdeling Package (EXOMOP) are shown as a solid red line. The model predicted ingress and egress points from EXOMOP are shown as dashed red vertical lines. The residuals (light curve - EXOMOP model) are shown in the second panel. See Table 1 for the cadence, out-of-transit RMS flux, and residual RMS flux for each light curve. The data points for all the transits are available in electronic form (see Table 2).

\section{LIGHT CURVE ANALYSIS}

To find the best fit to the light curves, we use the EXOplanet MOdeling Package (EXOMOP; Pearson et al. 2014; Turner et al. 2016b), ${ }^{3}$ which utilizes the analytic equations of Mandel \& Agol (2002) to generate a model transit. For a complete description of EXOMOP,

\footnotetext{
${ }^{3}$ EXOMOPv7.0 is used in the analysis and is available on Github at
} https://github.com/astrojake/EXOMOP. see Pearson et al. (2014) and Turner et al. (2016b). The $\chi^{2}$-fitting statistic for the model light curve used in EXOMOP is

$\chi^{2}=\sum_{i=1}^{N_{\mathrm{pts}}}\left[\frac{f_{i}(\mathrm{obs})-f_{i}(\text { model })}{\sigma_{i}(\mathrm{obs})}\right]^{2}$

where $N_{\text {pts }}$ is the total number of data points (Table 1$), f_{i}($ obs) is the observed flux at time $i, \sigma_{i}$ (obs) is the error in the observed flux and $f_{i}$ (model) is the calculated model flux.

EXOMOP uses the following procedure to find a best fit to the data. A Levenberg-Marquardt (LM) non-linear least squares 

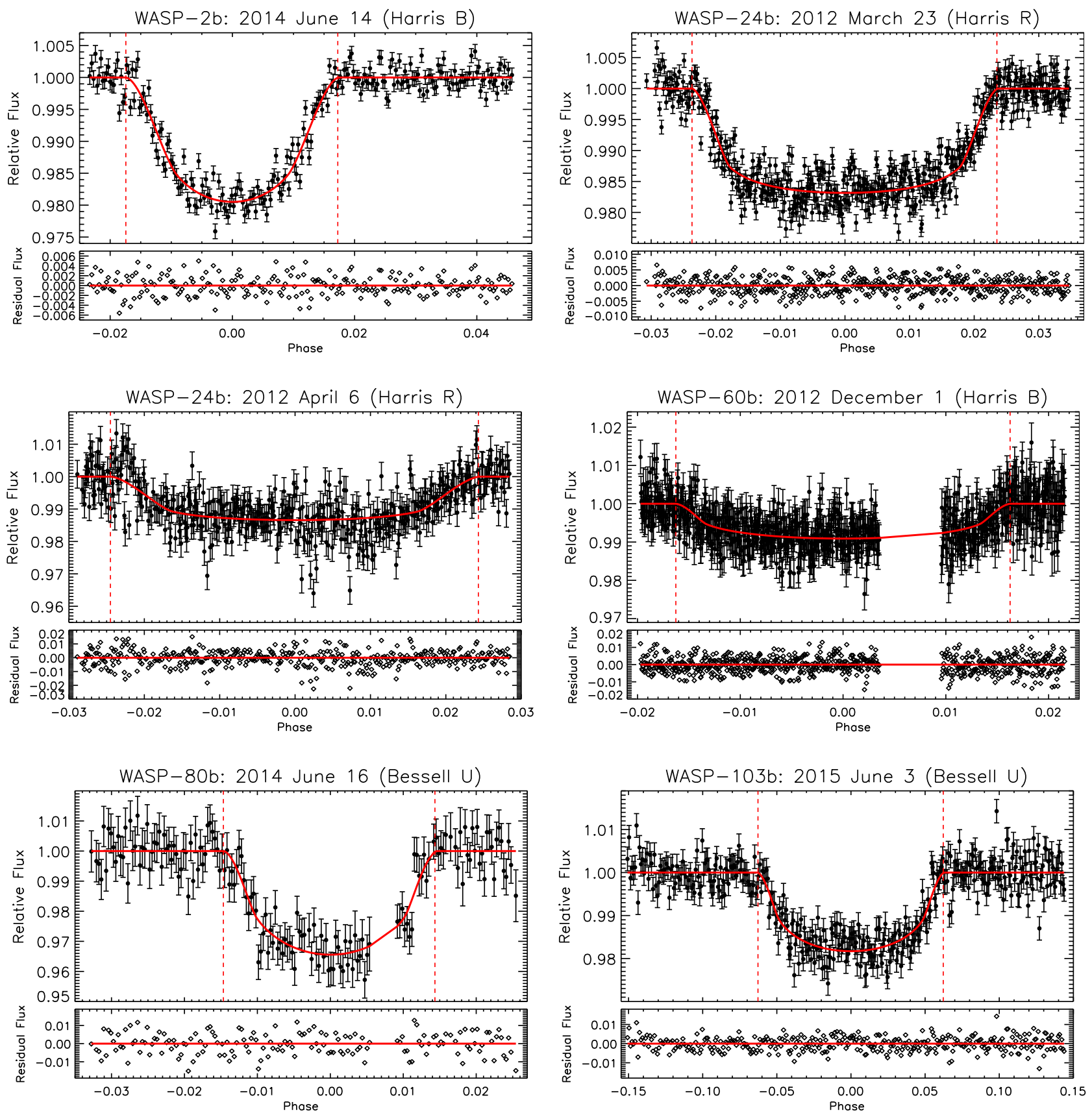

Figure 2. Light curves of WASP-2b, WASP-24b, WASP-60b, WASP-80b and WASP-103b. Other comments are the same as Fig. 1.

minimization (MPFIT; Markwardt 2009; Press et al. 1992) is performed on the data and a bootstrap Monte Carlo technique (Press et al. 1992) is used to calculate robust errors of the LM fitted parameters. Additionally, a Differential Evolution Markov Chain Monte Carlo (DE-MCMC; Braak 2006; Eastman, Gaudi \& Agol 2013) analysis is used to model the data. The fitted parameters that have the highest error bars from either the LM or DE-MCMC best-fitting model are used in the analysis. In every case, both models find results within $1 \sigma$ of each other. Additionally, EXOMOP uses the residual permutation (rosary bead; Southworth 2008), time-averaging (Pont, Zucker \& Queloz 2006) and wavelet (Carter \& Winn 2009) methods to assess the importance of red noise in both fitting methods. Not accounting for red noise in the data underestimates the fitted parameters (Pont et al. 2006; Carter \& Winn 2009). In order to be conservative, the red noise method which produces the largest errors is used to inflate the errors in the fitted parameters. Finally, in order to compensate for underestimated observational errors, we multiply the error bars of the fitted parameters by $\sqrt{\chi_{r}^{2}}$ when the reduced chi-squared $\left(\chi_{r}^{2}\right)$ of the data (Table 1) is greater than unity (e.g. Bruntt et al. 2006; Southworth, Wheatley \& Sams 2007a; Southworth, Bruntt \& Buzasi 2007b; Southworth 2008; Barnes et al. 2013; Turner et al. 2016b).

EXOMOP uses the Bayesian Information Criterion (BIC; Schwarz 1978) to assess over-fitting of the data. The BIC is defined as

$\mathrm{BIC}=\chi^{2}+k \ln N_{\mathrm{pts}}$, 


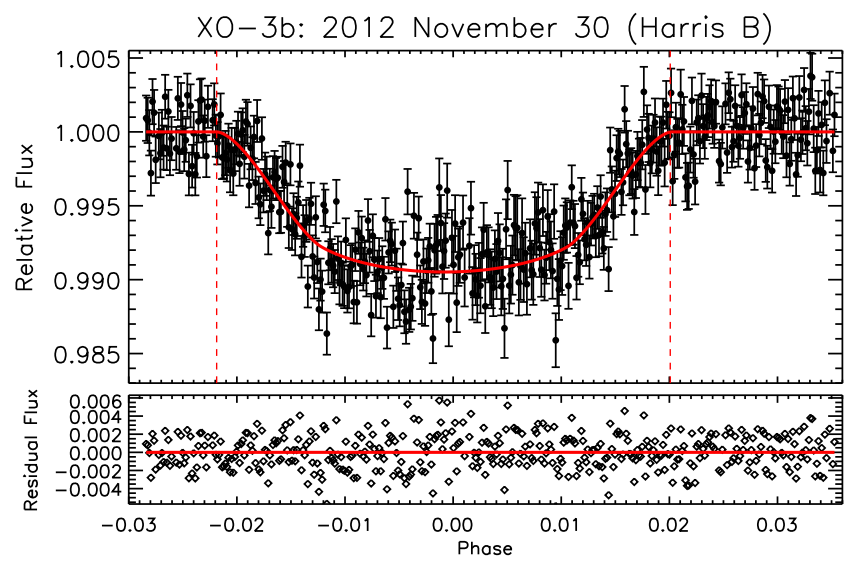

Figure 3. Light curve of XO-3b. Other comments are the same as Fig. 1.

where $\chi^{2}$ is calculated for the best-fitting model (equation 1) and $k$ is the number of free parameters (Table 1$)$ in the model fit $\left[f_{i}(\right.$ model $\left.)\right]$. The power of the BIC is the penalty for a higher number of fitted model parameters, making it a robust way to compare different best-fitting models. The preferred model is the one that produces the lowest BIC value.

Each transit is modelled with EXOMOP using 10000 iterations for the LM model and 20 chains and $20^{6}$ links for the DE-MCMC model. The Gelman-Rubin statistic (Gelman \& Rubin 1992) is used to ensure chain convergence (Ford 2006) in the MCMC model. During the analysis of each transit, the mid-transit time $\left(T_{c}\right)$, planetto-star radius $\left(R_{p} / R_{*}\right)$, scaled semi-major axis $\left(a / R_{*}\right)$ and inclination (i) are set as free parameters. The previously published values for $R_{p} / R_{*}, i$ and $a / R_{*}$ are used as priors for the LM model (Table 3). The results of the LM fit are used as the prior for the DE-MCMC. The eccentricity $(e)$, argument of periastron $(\omega)$, and period $\left(P_{p}\right)$ of each of the planets are fixed (see Table 3 for their values) in the analysis because these parameters have minimal effect on the overall shape of the light curve. The linear and quadratic limb darkening coefficients in each filter are taken from Claret \& Bloemen (2011) and interpolated to the stellar parameters of the host stars (see Table 4) using the EXOFAST applet ${ }^{4}$ (Eastman et al. 2013). In addition, a linear or quadratic least squares fit is modelled to the OoT baseline simultaneously with the Mandel \& Agol (2002) model. The BIC is used to determine whether to include any baseline fit in the best-fitting model and the baseline with the lowest BIC value is always chosen.

The light-curve parameters obtained from the EXOMOP analysis and the derived transit durations are summarized in Table 5. The modelled light curves can be found in Figs 1-3 and the physical parameters for our targets are derived as outlined in Section 4 (Tables 6 and 7). A thorough description of the modelling and results of each system can be found in Section 5 .

\section{PHYSICAL PROPERTIES OF THE SYSTEMS}

We use the results of our light-curve modelling with EXOMOP combined with other measurements in the literature to calculate the planetary mass (e.g. Winn 2011; Seager 2011), radius, density, surface gravity (e.g. Southworth et al. 2007a), modified equilibrium temperature (e.g. Southworth 2010), Safronov number (e.g. Safronov 1972; Southworth 2010) and atmospheric scaleheight (e.g. Seager 2011; de Wit \& Seager 2013). An updated period and ephemeris is also calculated and is described in detail in Section 4.1. To calculate the physical parameters we use the values from the modelling $\left(P_{p}\right.$, $R_{p} / R_{*}, i, a / R_{*}$ ), and for the orbital (e) and host star parameters (radial velocity amplitude, mass, radius, equilibrium temperature) we use the values found in the literature. When calculating the scaleheight, the mean molecular weight in the planet's atmosphere was set to 2.3 assuming an $\mathrm{H} / \mathrm{He}$-dominated atmosphere (de Wit \& Seager 2013). The physical parameters of all our systems can be found in Tables 6 and 7.

\subsection{Period determination}

By combining our mid-transit times found using EXOMOP with previously published mid-transit times, we can refine the orbital period of the targets. When necessary, the mid-transit times were transformed from HJD, which is based on UTC time, into BJD, which is based on Barycentric Dynamical Time (TDB), using the online converter ${ }^{5}$ by Eastman, Siverd \& Gaudi (2010). A refined ephemeris for each target is found by performing a weighted linear least-squares analysis using the following equation:

$T_{c}=T_{c}(0)+P_{p} \times E$,

where $T_{c}(0)$ is the mid-transit time at the discovery epoch measured in $\mathrm{BJD}_{\mathrm{TDB}}, P_{p}$ is the orbital period of the target and $E$ is the integer number of cycles after their discovery paper. See Tables 6 and 7 for an updated $T_{c}$ and $P_{b}$ for each system.

For every system, we also made observation minus calculation mid-transit time $(\mathrm{O}-\mathrm{C})$ plots in order to search for any TTVs due to other bodies in the system. We used the derived period and ephemeris found in Tables 6 and 7 and equation (3) for the calculated mid-transit times. The $\mathrm{O}-\mathrm{C}$ plots can be found in Figs 4 and 5 . The transit timing analysis for all our targets can be found in Table 8 (the entire table can be found online). We do not observe any significant TTVs in our data with the exception of a $3.8 \sigma$ deviation for WASP$80 \mathrm{~b}$ for our observed transit. Since the possible TTV is only one data point and may be caused by an unknown systematic error, more observations of WASP-80b are needed to confirm this result.

\section{INDIVIDUAL SYSTEMS}

\subsection{CoRoT-12b}

CoRoT-12b was discovered by the CoRoT satellite (Carone et al. 2012) and was confirmed by follow-up photometry and radialvelocity measurements (Gillon et al. 2010). CoRoT-12b is an inflated hot Jupiter with a low density which is well predicted by standard models (Fortney, Marley \& Barnes 2007) for irradiated planets (Gillon et al. 2010).

We observed a transit of CoRoT-12b on 2013 February 15 with the Harris $R$ filter (Fig. 1). We find an $R_{p} / R_{*}$ value $4.6 \sigma$ greater than the discovery value. Our derived physical parameters are in good agreement with Gillon et al. (2010). We find a planetary radius within $1.3 \sigma$ of the previously calculated value and a planetary mass within $1 \sigma$ (Tables 5 and 6). 
Table 2. Photometry of all our light curves.

\begin{tabular}{|c|c|c|c|c|c|c|c|}
\hline Planet name & Filter & Time (HJDutC) & Relative flux & Error bars & CCD X-Pos & CCD Y-Pos & Median airmass \\
\hline CoRoT-12b & Harris- $R$ & 2456338.584736 & 0.99648 & 0.003327 & 529.411 & 635.689 & 1.513072 \\
\hline CoRoT-12b & Harris- $R$ & 2456338.585434 & 0.998034 & 0.003213 & 527.611 & 634.955 & 1.507972 \\
\hline CoRoT-12b & Harris- $R$ & 2456338.586133 & 0.998494 & 0.003108 & 528.101 & 634.46 & 1.502944 \\
\hline CoRoT-12b & Harris- $R$ & 2456338.586831 & 0.99549 & $0.003 \quad 094$ & 528.858 & 634.639 & 1.497979 \\
\hline
\end{tabular}

Notes. This table is available in its entirety in machine-readable form in the online journal. A portion is shown here for guidance regarding its form and content.

Table 3. Parameters fixed for the light-curve fitting using EXOMOP.

\begin{tabular}{|c|c|c|c|c|c|c|}
\hline Planet & $\begin{array}{l}\text { Period }\left(P_{p}\right) \\
\text { (d) }\end{array}$ & $\mathrm{a} / R_{*}^{a}$ & $\begin{array}{c}\text { Inclination }(i)^{a} \\
\left(^{\circ}\right)\end{array}$ & Eccentricity $(e)$ & $\begin{array}{c}\text { Omega }(\omega) \\
\left(^{\circ}\right)\end{array}$ & Source \\
\hline CoRoT-12b & 2.828042 & 7.7402 & 85.48 & 0.070 & 105 & 1 \\
\hline HAT-P-5b & 2.788491 & 7.5 & 86.75 & 0 & 0 & 2 \\
\hline HAT-P-12b & 3.213089 & 11.7371 & 89.915 & 0 & 0 & 3 \\
\hline HAT-P-33b & 3.474474 & 6.56 & 87.2 & 0.148 & 96 & 4 \\
\hline HAT-P-37b & 2.797436 & 9.32 & 86.9 & 0.058 & 164 & 5 \\
\hline WASP-2b & $2.152218 \quad 12$ & 8.06 & 84.89 & 0 & 0 & 6 \\
\hline WASP-24b & 2.341213 & 5.98 & 83.64 & 0 & 0 & 7 \\
\hline WASP-60b & 4.3050011 & 10 & 87.9 & 0 & 0 & 8 \\
\hline WASP-80b & 3.06785 & 12.989 & 89.92 & 0.07 & 0 & 9 \\
\hline WASP-103b & 0.925542 & 2.978 & 86.3 & 0 & 0 & 10 \\
\hline $\mathrm{XO}-3 \mathrm{~b}$ & 3.191524 & 7.07 & 84.2 & 0.26 & 345.8 & 11 \\
\hline
\end{tabular}

${ }^{a}$ These parameter values were not fixed in the final analysis but were used as priors for the MCMC.

References. (1) Gillon et al. (2010), (2) Bakos et al. (2007), (3) Lee et al. (2012), (4) Hartman et al. (2011), (5) Bakos et al. (2012), (6) Becker et al. (2013), (7) Street et al. (2010), (8) Hébrard et al. (2013), (9) Triaud et al. (2013), (10) Gillon et al. (2014), (11) Winn et al. (2008).

Table 4. Limb darkening coefficients for the light-curve fitting using EXOMOP.

\begin{tabular}{|c|c|c|c|c|c|c|c|}
\hline Planet & Filter & Linear coefficient ${ }^{a}$ & Quadratic coefficient ${ }^{a}$ & $T_{\text {eff }}[\mathrm{K}]$ & {$[\mathrm{Fe} / \mathrm{H}]$} & $\log g[\mathrm{cgs}]$ & Source \\
\hline CoRoT-12b & $R$ & 0.39440901 & 0.26682249 & 5675 & 0.160 & 4.375 & 1 \\
\hline HAT-P-5b & $U$ & 0.75552025 & 0.093484300 & 5960 & 0.240 & 4.368 & 2 \\
\hline HAT-P-12b & $B$ & 0.93774724 & -0.083432883 & 4650 & -0.290 & 4.610 & 3 \\
\hline HAT-P-33b & $R$ & 0.27628872 & 0.32295169 & 6401 & 0.05 & 4.15 & 4 \\
\hline HAT-P-37b & $B$ & 0.72832760 & 0.097543998 & 5500 & 0.03 & 4.52 & 5 \\
\hline HAT-P-37b & $R$ & 0.41967640 & 0.25020840 & 5500 & 0.03 & 4.52 & 5 \\
\hline WASP-2b & $B$ & 0.82272126 & 0.018632333 & 5200 & 0.100 & 4.537 & 2 \\
\hline WASP-24b & $R$ & 0.31410756 & 0.30624587 & 6080 & -0.002 & 4.26 & 6 \\
\hline WASP-60b & $B$ & 0.61455358 & 0.18477201 & 5900 & -0.040 & 4.20 & 7 \\
\hline WASP-80b & $U$ & 0.82663825 & -0.029831771 & 4150 & -0.140 & 4.60 & 8 \\
\hline WASP-103b & $U$ & 0.65536932 & 0.17875591 & 6110 & 0.060 & 4.22 & 9 \\
\hline $\mathrm{XO}-3 \mathrm{~b}$ & $B$ & 0.50449954 & 0.25897027 & 6429 & -0.177 & 3.950 & 10 \\
\hline
\end{tabular}

${ }^{a}$ The limb darkening coefficients are taken from Claret \& Bloemen (2011) and interpolated to the stellar parameters of their host star using EXOFAST. References. (1) Gillon et al. (2010), (2) Torres, Winn \& Holman (2008), (3) Hartman et al. (2009), (4) Hartman et al. (2011), (5) Bakos et al. (2012), (6) Street et al. (2010), (7) Hébrard et al. (2013), (8) Triaud et al. (2013), (9) Gillon et al. (2014), (10) Johns-Krull et al. (2008).

\subsection{HAT-P-5b}

HAT-P-5b is a hot Jupiter discovered by the HATNet project which orbits a slightly metal-rich star (Bakos et al. 2007). Follow-up multicolour transit observations of HAT-P-5b by Southworth et al. (2012) confirmed the existence of the planet and searched for a variation in planetary radius with wavelength. A significantly larger radius was found in the $U$-band than expected from Rayleigh scattering alone, which the authors suggest may be due to an unknown systematic error.

We observed a transit of HAT-P-5b on 2015 June 6 with the Bessell $U$ filter (Fig. 1). Our derived physical parameters are in agreement with previous literature (Tables 5 and 6). We derive a $U$-band radius consistent with a weighted average of radii taken from 350-733 nm within $1 \sigma$ (Table 9; Fig. 6). The error on our
$U$-band observation is too large to determine if the observation by Southworth et al. (2012) in the same band may have an unknown systematic error (as suggested by them). Our calculated period is in good agreement with the value found by Southworth et al. (2012) with a similar uncertainty.

\subsection{HAT-P-12b}

HAT-P-12b is a low density, sub-Saturn mass planet discovered by the HAT survey (Hartman et al. 2009). Multiple photometric studies have further refined the system's parameters and searched for TTVs (Sada et al. 2012; Sokov et al. 2012; Lee et al. 2012; Line et al. 2013; Mallonn et al. 2015b; Sing et al. 2016; Sada \& Ramón-Fox 2016). Sing et al. (2016) find a strong optical scattering slope from blue 
Table 5. Light-curve parameters derived in this study using EXOMOP.

\begin{tabular}{|c|c|c|c|c|c|}
\hline Planet & CoRoT-12b & HAT-P-5b & HAT-P-12b & HAT-P-33b & HAT-P-37b \\
\hline Date & 2013 February 15 & 2015 June 6 & 2014 January 19 & 2012 April 6 & 2015 July 1 \\
\hline Filter $^{a}$ & $R$ & $U$ & $B$ & $R$ & B \\
\hline$T_{c}\left(\mathrm{BJD}_{\mathrm{TDB}}-2450000\right)$ & $6338.67097 \pm 0.00074$ & $7180.82658 \pm 0.00076$ & $6677.97482 \pm 0.00047$ & $6024.71746 \pm 0.0012$ & $7205.91376 \pm 0.00054$ \\
\hline $\mathrm{R}_{p} / \mathrm{R}_{*}$ & $0.1645_{-0.0040}^{+0.0038}$ & $0.1225 \pm 0.0051$ & $0.1386_{-0.0014}^{+0.0013}$ & $0.1152 \pm 0.0017$ & $0.1253 \pm 0.0021$ \\
\hline$a / R_{*}$ & $6.59_{-0.29}^{+0.31}$ & $6.05 \pm 0.44$ & $11.86 \pm 0.57$ & $5.67 \pm 0.13$ & $10.82 \pm 0.91$ \\
\hline Inclination $\left(^{\circ}\right)$ & $83.54 \pm 0.71$ & $83.31 \pm 1.11$ & $90.98 \pm 1.09$ & $90.08 \pm 3.43$ & 89.991 .83 \\
\hline Duration (mins) & $174.0 \pm 1.4$ & $184.1 \pm 1.9$ & $139.8 \pm 4.4$ & $270.45 \pm 0.48$ & $132.8 \pm 2.7$ \\
\hline Red noise (mmag) & 0.0001 & 1.60 & 0.21 & 0.78 & 0.0001 \\
\hline OoT baseline function & None & None & Quadratic & None & None \\
\hline Planet & HAT-P-37b & HAT-P-37b & WASP-2b & WASP-24b & WASP-24b \\
\hline Date & 2015 July 1 & Weighted average & 2014 June 14 & 2012 March 23 & 2012 April 6 \\
\hline Filter $^{a}$ & $R$ & - & $B$ & $R$ & $R$ \\
\hline$T_{c}\left(\mathrm{BJD}_{\mathrm{TDB}}-2450000\right)$ & $7205.91325 \pm 0.00056$ & - & $6823.83839 \pm 0.00055$ & $6010.8437 \pm 0.0017$ & $6024.8910 \pm 0.0015$ \\
\hline $\mathrm{R}_{p} / \mathrm{R}_{*}$ & $0.1361 \pm 0.0028$ & $0.1291 \pm 0.0017$ & $0.1383 \pm 0.0049$ & $0.1139 \pm 0.0015$ & $0.1113 \pm 0.0043$ \\
\hline$a / R_{*}$ & $9.14 \pm 0.63$ & $9.68 \pm 0.52$ & $8.05 \pm 1.21$ & $7.42 \pm 0.15$ & $6.06 \pm 0.73$ \\
\hline Inclination $\left(^{\circ}\right)$ & $86.73 \pm 0.93$ & $87.4 \pm 0.82$ & $84.86 \pm 1.61$ & $90.0 \pm 5.4$ & $83.95 \pm 2.74$ \\
\hline Duration (mins) & $140.5 \pm 2.7$ & $136.6 \pm 1.9$ & $108.4 \pm 1.4$ & $159.5 \pm 0.6$ & $165.0 \pm 0.6$ \\
\hline Red noise (mmag) & 0.34 & - & 0.00 & 0.27 & 0.60 \\
\hline OoT baseline function & None & - & Quadratic & Quadratic & Linear \\
\hline Planet & WASP-24b & WASP-60b & WASP-80b & WASP-103b & $\mathrm{XO}-3 \mathrm{~b}$ \\
\hline Date & Weighted average & 2012 December 1 & 2014 June 16 & 2015 June 3 & 2012 November 30 \\
\hline Filter $^{a}$ & $R$ & $B$ & $U$ & $U$ & $B$ \\
\hline$T_{c}\left(\mathrm{BJD}_{\mathrm{TDB}}-2450000\right)$ & - & $6263.6330 \pm 0.0012$ & $6824.88661 \pm 0.00091$ & $7177.8222_{-0.0009}^{+0.0015}$ & $6262.6566 \pm 0.0015$ \\
\hline $\mathrm{R}_{p} / \mathrm{R}_{*}$ & $0.1136 \pm 0.0014$ & $0.0852 \pm 0.0036$ & $0.1615 \pm 0.0033$ & $0.1181 \pm 0.0016$ & $0.0968 \pm 0.0023$ \\
\hline$a / R_{*}$ & $7.36 \pm 0.15$ & $9.49 \pm 1.81$ & $12.85 \pm 0.42$ & $2.90 \pm 0.05$ & $5.68 \pm 0.51$ \\
\hline Inclination $\left(^{\circ}\right)$ & $85.19 \pm 2.44$ & $87.48 \pm 2.83$ & $90.0 \pm 1.8$ & $90.00 \pm 0.18$ & $81.75 \pm 0.77$ \\
\hline Duration (mins) & $162.1 \pm 0.4$ & $201.9 \pm 0.3$ & $126.7 \pm 2.2$ & $167.6 \pm 1.5$ & $185.4 \pm 0.9$ \\
\hline Red noise (mmag) & - & 0.00 & 0.01 & 0.001 & 0.001 \\
\hline OoT baseline function & - & None & Quadratic & Linear & None \\
\hline
\end{tabular}

${ }^{a}$ Filter: $U$ is the Bessell $U(303-417 \mathrm{~nm}), B$ is the Harris $B(330-550 \mathrm{~nm})$ and $R$ is the Harris $R(550-900 \mathrm{~nm})$.

to near-IR wavelengths using Hubble Space Telescope and Spitzer Space Telescope transmission spectrum data.

We observed a transit of HAT-P-12b on 2014 January 19 using the Harris $B$ filter (Fig. 1). We derive an optical $R_{p} / R_{*}$ within $1 \sigma$ of previously derived radii at optical wavelengths (Table 9; Fig. 6). These results are consistent with the planet having high clouds in its atmosphere (e.g. Seager \& Sasselov 2000; Kreidberg et al. 2014) and the finding by Line et al. (2013) that HAT-P-12b has a cloudy atmosphere. We also find a period similar to Mallonn et al. (2015b).

\subsection{HAT-P-33b}

HAT-P-33b is an inflated hot Jupiter orbiting a high-jitter star (Hartman et al. 2011). The high-jitter is believed to be caused by convective inhomogeneities in the host star (Saar, Butler \& Marcy 1998; Hartman et al. 2011). The planetary radius and mass, which both depend on eccentricity, and the stellar parameters are not well constrained due to the large jitter $\left(20 \mathrm{~m} \mathrm{~s}^{-1}\right)$. HAT-P-33b's radius is either 1.7 or $1.8 R_{\text {Jup }}$ assuming a circular or eccentric orbit, respectively. The first follow-up observations by the Transiting Exoplanet Monitoring Project (TEMP) of HAT-P-33b confirmed the discovery parameters and detected no signs of TTVs (Wang et al. 2017).

We observed one transit of HAT-P-33b on 2012 April 6 with the Harris $R$ filter (Fig. 1). We find an $R$-band $R_{p} / R_{*}$ value which is larger by $3.4 \sigma$ from the discovery $R_{p} / R_{*}$ (Table 9 ). Follow-up observations are needed to determine the cause of this discrepancy.

\subsection{HAT-P-37b}

HAT-P-37b was identified by the HATNet survey and was confirmed by high-resolution spectroscopy and further photometric observations (Bakos et al. 2012). HAT-P-37b is a hot Jupiter with a planetary mass of $1.169 \pm 0.103 M_{\text {Jup }}$, a radius of $1.178 \pm 0.077 R_{\mathrm{Jup}}$, and a period of $2.797436 \pm 0.000007 \mathrm{~d}$. Additional follow-up observations by Maciejewski et al. (2016) confirmed these planetary parameters.

We obtained two transits of HAT-P-37b on 2015 July 1 with the Harris $B$ and $R$ filters (Fig. 1). We derive an $R_{p} / R_{*}$ for each filter which differ by $1.7 \sigma$, with a larger radius in the $R$ band (Table 9; Fig. 6). The $B$-band $R_{p} / R_{*}$ is smaller by $2.85 \sigma$ from the near-IR $R_{p} / R_{*}$ (Table 9; Bakos et al. 2012). Our derived $R$-band $R_{p} / R_{*}$ value agrees within $1 \sigma$ of the Sloan $i$ band value obtained by Bakos et al. (2012). Near-UV observations are needed to determine if the slope between the $B$ and $R$ filters is real or an unknown systematic in the data. Our other derived physical parameters agree with previous literature to within $1 \sigma$ (Tables 5 and 6 ). We also calculate a refined period with a factor of 6 decrease in error.

\subsection{WASP-2b}

WASP-2b is a short-period hot Jupiter discovered by the WASP survey and confirmed by radial-velocity measurements taken with the SOPHIE spectrograph (Collier Cameron et al. 2007). Extensive photometry and radial velocity measurements have been performed on WASP-2b, further refining its system parameters (Charbonneau et al. 2007; Daemgen et al. 2009; Southworth et al. 2010; 
Table 6. Physical parameters derived in this study for CoRoT-12b, HAT-P-5b, HAT-P-12b, HAT-P-33b, HAT-P-37b, WASP-2b, WASP-24b, WASP-60b and WASP-80b.

\begin{tabular}{|c|c|c|c|}
\hline Planet & CoRoT-12b & HAT-P-5b & HAT-P-12b \\
\hline Date & 2013 February 15 & 2015 June 6 & 2014 January 19 \\
\hline Period (d) & $2.828051 \pm 0.000080$ & $2.78847280 \pm 0.00000039$ & $3.21305761 \pm 0.00000020$ \\
\hline$T_{c}(0)(\mathrm{BJD}-2450000)$ & $4398.628 \pm 0.055$ & $4241.77716 \pm 0.00015$ & $4187.85623 \pm 0.00013$ \\
\hline$M_{b}\left(M_{\mathrm{Jup}}\right)$ & $0.922 \pm 0.072$ & $1.06 \pm 0.12$ & $0.211 \pm 0.012$ \\
\hline Our $R_{b}\left(R_{\mathrm{Jup}}\right)$ & $1.79 \pm 0.15$ & $1.36 \pm 0.057$ & $0.949 \pm 0.017$ \\
\hline Reference $R_{b}\left(R_{\mathrm{Jup}}\right)$ & $1.44 \pm 0.13(\mathrm{a})$ & $1.26 \pm 0.05(b)$ & $0.959 \pm 0.029(\mathrm{c})$ \\
\hline$\rho_{b}(\mathrm{cgs})$ & $0.200 \pm 0.054$ & $0.531 \pm 0.088$ & $0.306 \pm 0.023$ \\
\hline $\log g_{b}(\mathrm{cgs})$ & $2.72 \pm 0.12$ & $2.946 \pm 0.085$ & $2.77 \pm 0.052$ \\
\hline$T_{\mathrm{eq}}^{\prime}(\mathrm{K})$ & $1563 \pm 22$ & $1713 \pm 29$ & $954 \pm 12$ \\
\hline$\Theta$ & $0.0327 \pm 0.0054$ & $0.0431 \pm 0.0064$ & $0.0235 \pm 0.0019$ \\
\hline$a(\mathrm{au})$ & $0.0342 \pm 0.0032$ & $0.0320 \pm 0.0023$ & $0.0386 \pm 0.0019$ \\
\hline$H(\mathrm{~km})$ & $1521 \pm 402$ & $979 \pm 193$ & $822 \pm 98$ \\
\hline Planet & HAT-P-33b & HAT-P-37b & WASP-2b \\
\hline Date & 2012 April 6 & 2015 July 1 & 2014 June 14 \\
\hline Period (d) & $3.4744750 \pm 0.00000037$ & $2.79744149 \pm 0.00000083$ & $2.152 \quad 221 \quad 14 \pm 0.00000019$ \\
\hline$T_{c}(0)(\mathrm{BJD}-2450000)$ & $5110.92726 \pm 0.00012$ & $5616.96710 \pm 0.00028$ & $3991.515553 \pm 0.000074$ \\
\hline$M_{b}\left(M_{\mathrm{Jup}}\right)$ & $1.26 \pm 0.23$ & $1.17 \pm 0.10$ & $0.880 \pm 0.087$ \\
\hline Our $R_{b}\left(R_{\mathrm{Jup}}\right)$ & $1.99 \pm 0.32$ & $1.16 \pm 0.06$ & $1.12 \pm 0.13$ \\
\hline Reference $R_{b}\left(R_{\mathrm{Jup}}\right)$ & $1.827 \pm 0.290(\mathrm{~d})$ & $1.178 \pm 0.077(\mathrm{e})$ & $1.043 \pm 0.033$ (f) \\
\hline$\rho_{b}(\mathrm{cgs})$ & $0.20 \pm 0.10$ & $0.93 \pm 0.17$ & $0.77 \pm 0.28$ \\
\hline $\log g_{b}(\mathrm{cgs})$ & $2.83 \pm 0.21$ & $3.369 \pm 0.088$ & $3.25 \pm 0.18$ \\
\hline$T_{\text {eq }}^{\prime}(\mathrm{K})$ & $1901 \pm 26$ & $1250 \pm 22$ & $1284 \pm 20$ \\
\hline$\Theta$ & $0.042 \pm 0.013$ & $0.085 \pm 0.012$ & $0.058 \pm 0.016$ \\
\hline$a(\mathrm{au})$ & $0.0468 \pm 0.0075$ & $0.0394 \pm 0.0029$ & $0.0312 \pm 0.0056$ \\
\hline$H(\mathrm{~km})$ & $1408 \pm 679$ & $270 \pm 54$ & $364 \pm 155$ \\
\hline Planet & WASP-24b & WASP-60b & WASP-80b \\
\hline Date & Combined & 2012 December 1 & 2014 June 16 \\
\hline Period (d) & $2.34121877 \pm 0.00000030$ & $4.305022 \pm 0.000021$ & $3.06785925 \pm 0.00000047$ \\
\hline$T_{c}(0)(\mathrm{BJD}-2450000)$ & $4945.589444 \pm 0.000090$ & $5747.0302 \pm 0.0022$ & $6125.418034 \pm 0.000052$ \\
\hline$M_{b}\left(M_{\mathrm{Jup}}\right)$ & $1.032 \pm 0.037$ & $0.512 \pm 0.034$ & $0.551 \pm 0.036$ \\
\hline Our $R_{b}\left(R_{\mathrm{Jup}}\right)$ & $1.27 \pm 0.055$ & $0.94 \pm 0.12$ & $0.99 \pm 0.24$ \\
\hline Reference $R_{b}\left(R_{\mathrm{Jup}}\right)$ & $1.303 \pm 0.047(\mathrm{~g})$ & $0.86 \pm 0.12(\mathrm{~h})$ & $0.999 \pm 0.031$ (i) \\
\hline$\rho_{b}(\mathrm{cgs})$ & $0.628 \pm 0.085$ & $0.75 \pm 0.27$ & $0.71 \pm 0.51$ \\
\hline $\log g_{b}(\mathrm{cgs})$ & $3.279 \pm 0.057$ & $3.11 \pm 0.22$ & $3.22 \pm 0.29$ \\
\hline$T_{\mathrm{eq}}^{\prime}(\mathrm{K})$ & $1583 \pm 27$ & $1354 \pm 23$ & $817 \pm 20$ \\
\hline$\Theta$ & $0.0566 \pm 0.0043$ & $0.051 \pm 0.013$ & $0.072 \pm 0.025$ \\
\hline$a(\mathrm{au})$ & $0.0392 \pm 0.0018$ & $0.050 \pm 0.011$ & $0.0376 \pm 0.0090$ \\
\hline$H(\mathrm{~km})$ & $421 \pm 55$ & $536 \pm 274$ & $248 \pm 168$ \\
\hline
\end{tabular}

References. (a) Gillon et al. (2010); (b) Bakos et al. (2007); (c) Hartman et al. (2009); (d) Hartman et al. (2011); (e) Bakos et al. (2012); (f) Southworth et al. (2010); (g) Southworth et al. (2014); (h) Hébrard et al. (2013); (i) Triaud et al. (2015).

Table 7. Physical parameters derived in this study for WASP-103b and XO-3b.

\begin{tabular}{lcc}
\hline Planet & WASP-103b & XO-3b \\
\hline Date & 2015 June 3 & 2012 November 30 \\
Period (d) & $0.9255454 \pm 0.0000010$ & $3.19153125 \pm 0.00000053$ \\
$T_{c}(0)($ BJD-2450000) & $6459.59948 \pm 0.00041$ & $2997.72200 \pm 0.00040$ \\
$M_{b}\left(M_{\text {Jup }}\right)$ & $1.484 \pm 0.082$ & $13.07 \pm 0.66$ \\
Our $R_{b}\left(R_{\text {Jup }}\right)$ & $1.640 \pm 0.066$ & $1.403 \pm 0.093$ \\
Reference $R_{b}\left(R_{\text {Jup }}\right)$ & $1.528 \pm 0.073(\mathrm{a})$ & $1.217 \pm 0.073(\mathrm{~b})$ \\
$\rho_{b}(\mathrm{cgs})$ & $0.417 \pm 0.055$ & $5.87 \pm 1.20$ \\
$\log g_{b}(\mathrm{cgs})$ & $3.114 \pm 0.055$ & $4.05 \pm 0.11$ \\
$T_{\text {eq }}^{\prime}(\mathrm{K})$ & $2537 \pm 42$ & $2011 \pm 13$ \\
$\Theta$ & $0.0286 \pm 0.0023$ & $0.519 \pm 0.075$ \\
$a(\mathrm{au})$ & $0.09361 \pm 0.00078$ & $0.0393 \pm 0.0041$ \\
$H(\mathrm{~km})$ & $986 \pm 125$ & $90 \pm 22$ \\
\hline
\end{tabular}

References. (a) Gillon et al. (2014); (b) Winn et al. (2008). 

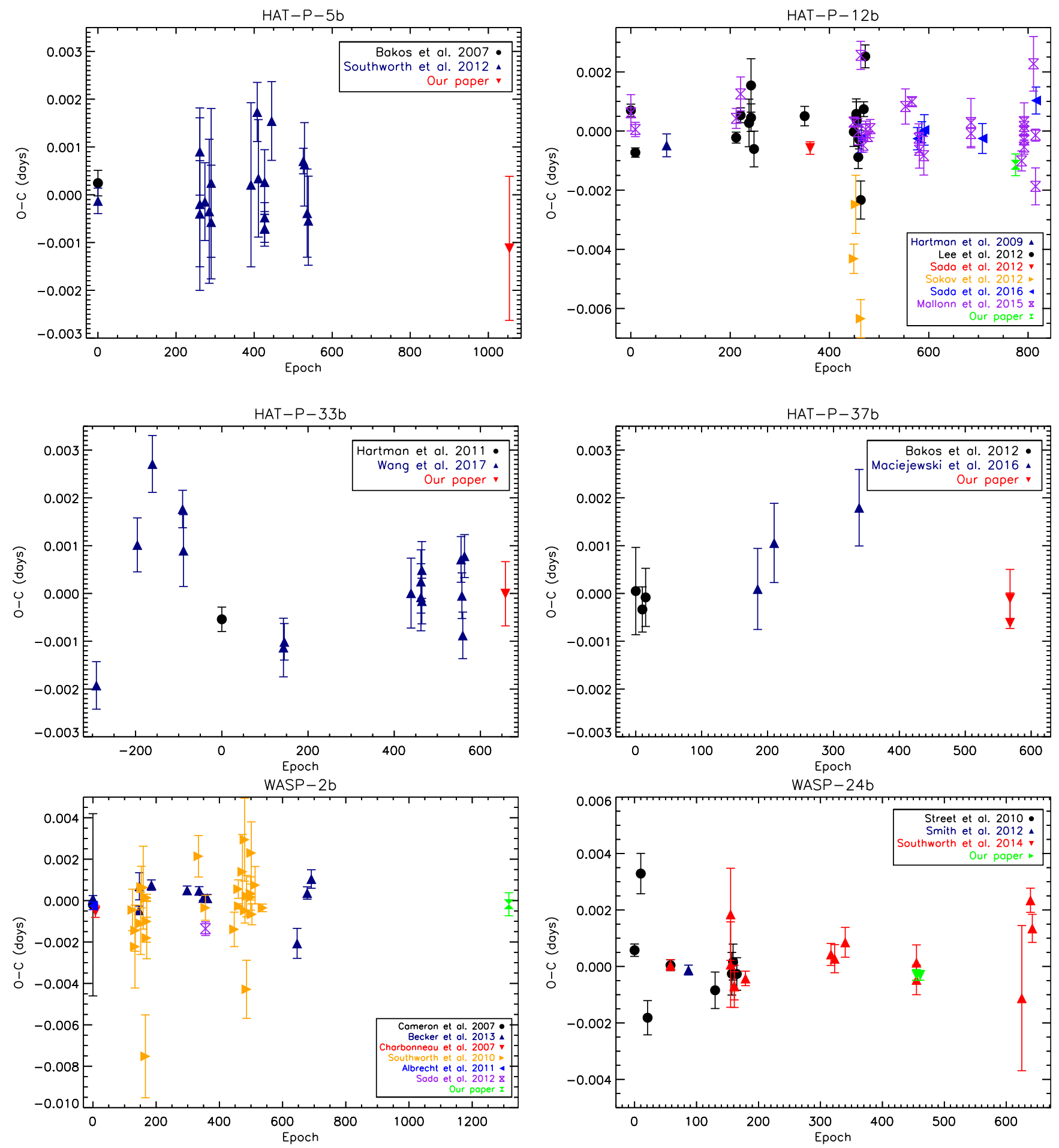

Figure 4. Observation minus calculation mid-transit time (O-C) plots of HAT-P-5b, HAT-P-12b, HAT-P-33b, HAT-P-37b, WASP-2b and WASP-24b from this paper and previous literature. We do not see any evidence for TTVs.

Triaud et al. 2010; Albrecht et al. 2011; Zhang et al. 2011; Husnoo et al. 2012; Sada et al. 2012; Becker et al. 2013).

We observed WASP-2b on 2014 June 14 with the Harris $B$ filter (Fig. 2). Our derived physical parameters and transit depth agree with previous literature to within $1 \sigma$ and we calculate a period with a factor of 2 decrease in error (Tables 5 and 6 ).

\subsection{WASP-24b}

WASP-24b is a hot Jupiter detected by WASP and confirmed by radial velocity measurements and additional photometric observations (Street et al. 2010). Further photometric studies calculated improved system parameters (Southworth et al. 2014), and radial velocity measurements were used to determine that the planet exhibits 

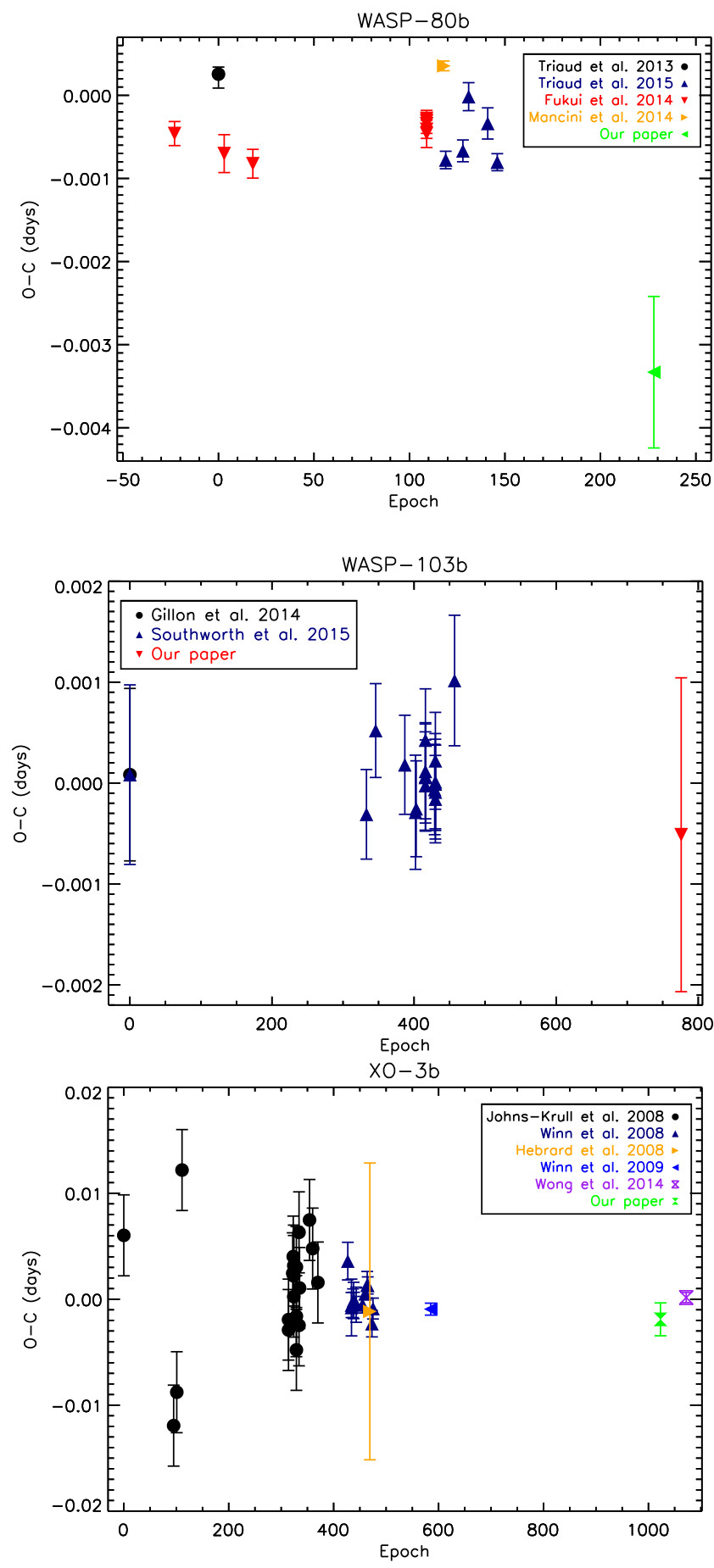

Figure 5. O-C plot of WASP-80b, XO-3b and WASP-103b from this paper and previous literature. We do not see any TTVs with the exception of WASP-80b but there are large uncertainties in our measurement, so we recommend follow-up observations to verify this claim.

a symmetrical Rossiter-McLaughlin effect, indicating a prograde, well-aligned orbit (Simpson et al. 2011).

Our observations of WASP-24b took place on 2012 March 23 and 2012 April 6 (Fig. 2). We obtained two transits with the Harris $R$ filter and each transit was modelled separately. The $R_{p} / R_{*}$ of both dates overlap each other within $1 \sigma$. We then found the weighted average of the light-curve parameters before deriving the physical parameters. Our weighted average $R_{p} / R_{*}$ disagrees with previous
$R$-band observations (Southworth et al. 2014) by $4 \sigma$ (Table 9; Fig. 6). The cause of this difference is unknown but future observations can put better constraints on transit depth and solve this discrepancy. Our other derived parameters generally agree with previous results except for our planetary radius and equilibrium temperature, which differ from Southworth et al. (2014) by $1.4 \sigma$ and $1.9 \sigma$, respectively (Tables 5 and 6 ). We calculate a new period with a factor of 2.6 decrease in error (Table 6).

\subsection{WASP-60b}

WASP-60b was identified by WASP-North and was confirmed by radial-velocity measurements and follow-up photometry (Hébrard et al. 2013). WASP-60b is an unexpectedly compact planet orbiting a metal-poor star.

We observed a transit of WASP-60b with the Harris $B$ filter on 2012 December 1 (Fig. 2). This observation is the first follow-up light curve of WASP-60b. During observations, the automatic guider briefly failed, resulting in a hole in the transit light curve. Despite this, we are able to derive parameters that agree with previous literature to within $1 \sigma$ (Tables 5 and 6 ). We find a $B$-band $R_{p} / R_{*}$ value $1.3 \sigma$ greater than the discovery $R_{p} / R_{*}$.

\subsection{WASP-80b}

WASP-80b is a warm Saturn/hot Jupiter $\left(M_{p}=0.55 \pm 0.04 M_{\text {jup }}\right)$ with one of the largest transit depths $(0.17126 \pm 0.00031)$ discovered so far (Triaud et al. 2013). Multiple photometric studies have been done at various wavelengths to refine WASP-80b's planetary parameters (Fukui et al. 2014; Mancini et al. 2014; Triaud et al. 2015; Salz et al. 2015; Sedaghati et al. 2017; Parviainen et al. 2017). The planet has a transmission spectrum consistent with thick clouds and atmospheric haze (Fukui et al. 2014).

We observed WASP-80b on 2014 June 16 with the Bessell $U$ filter, obtaining one transit (Fig. 2). Inclement weather conditions caused our guider to briefly fail, resulting in a hole in the transit light curve. We derive physical parameters that closely agree with previous literature and also calculate a slightly a refined period with a factor of 2 decrease in error (Tables 5 and 6). Our observations possibly detect a TTV compared to previous work by $3.7 \sigma$ (Section 4.1), however, further observations of WASP-80b are needed in order to confirm this result.

\subsection{WASP-103b}

WASP-103b is a hot Jupiter detected by the WASP survey with a mass of $1.49 \pm 0.09 M_{\text {jup }}$, short period planet $\left(P_{p}=0.925542 \pm\right.$ $0.000019 \mathrm{~d})$, and has an orbital radius only 20 per cent larger than its Roche radius (Gillon et al. 2014). It was found that there is a faint, cool and nearby (with a sky-projected separation of $0.242 \pm 0.016$ arcsec) companion star of WASP-103 (Wöllert \& Brandner 2015; Ngo et al. 2016). Further photometric observations were made to refine WASP-103b's planetary parameters and ephemeris (Southworth et al. 2015; Southworth \& Evans 2016; Lendl et al. 2017). A comparison of observed planetary radius at different wavelengths found a larger radius at bluer optical wavelengths, but Southworth \& Evans (2016) state that Rayleigh scattering cannot be the main cause even when including the contamination of the nearby companion star.

We observed WASP-103b on 2015 June 3 with the Bessell $U$ filter (Fig. 2). We derive a dilution-corrected near-UV $\left(R_{p} / R_{*}\right)_{\text {cor }}$ that differs from the discovery value by $2.1 \sigma$. Our other calculated 
Table 8. Results of the transit timing analysis.

\begin{tabular}{lllcccc}
\hline Planet name & $T_{c}\left(\mathrm{BJD}_{\mathrm{TDB}}\right)$ & $T_{c}$ error $(\mathrm{d})$ & Epoch & O-C $(\mathrm{d})$ & O-C error $(\mathrm{d})$ & Source \\
\hline CoRoT-12b & 2456338.67097 & 0.00074 & 686 & -0.0000012 & 0.055 & This paper \\
CoRoT-12b & 2454398.628 & 0.055 & 0 & 0.00000000046 & 0.078 & Gillon et al. (2010)
\end{tabular}

Notes. This table is available in its entirety in machine-readable form in the online journal. A portion is shown here for guidance regarding its form and content.

Table 9. $R_{p} / R_{*}$ and effective central wavelength $\lambda_{\text {eff }}$ from this paper and previous literature for all targets.

\begin{tabular}{llccc}
\hline Planet & Filter & Wavelength $(\mathrm{nm})$ & $R_{p} / R_{*}$ & Source \\
\hline CoRoT-12b & Harris $R$ & 658 & $0.1645 \pm 0.0040$ & This paper \\
CoRoT-12b & Clear & $400-900$ & $0.1321 \pm 0.0011$ & Gillon et al. (2010)
\end{tabular}

Notes. This table is available in its entirety in machine-readable form in the online journal. A portion is shown here for guidance regarding its form and content.

parameters agree with previous literature to within $1 \sigma$ and our calculated period closely agrees with the period found by Southworth et al. (2015) (Tables 5 and 7). A variation in $R_{p} / R_{*}$ is found from the ultraviolet to the near-infrared wavelengths (Table 9; Fig. 7) consistent with that found by Southworth \& Evans (2016).

We correct for the dilution due to the companion star being in our aperture using the procedure described below (this procedure is similar to that done by Southworth \& Evans 2016). (1) The light curve is modelled with ЕХОмоР and we find an uncorrected transit depth of $\left(R_{p} / R_{*}\right)_{\text {uncor }}=0.1174 \pm 0.0016$. (2) Theoretical spectra of both stars are produced using ATLAS9-ODFNEW (Castelli \& Kurucz 2004). For WASP-103, we use $T_{\text {eff }}=6110 \mathrm{~K}$ and $M_{\text {star }}=1.22 \mathrm{M}_{\odot}($ Gillon et al. 2014) and for the companion star, we use $T_{\text {eff }}=4405 \mathrm{~K}$ (Southworth \& Evans 2016) and $M_{\text {star }}=0.721 \mathrm{M}_{\odot} \quad(\mathrm{Ngo}$ et al. 2016). Additionally, in order to scale the spectrum correctly, we use the mass-luminosity relation $L=\mathrm{L}_{\odot}\left(M / \mathrm{M}_{\odot}\right)^{4}$ for stars between $0.5 \mathrm{M}_{\odot}$ and $2 \mathrm{M}_{\odot}$. (3) The ATLAS9-ODFNEW model spectra is convolved with the bandpass of the Bessell $U$ filter (Bessell 1979). (4) The corrected transit depth, $\left(R_{p} / R_{*}\right)_{\text {cor }}$, is found using the equation (Ciardi et al. 2015)

$$
\left(\frac{R_{p}}{R_{*}}\right)_{\text {cor }}=\left(\frac{R_{p}}{R_{*}}\right)_{\text {uncor }} \sqrt{\frac{F_{\text {tot }}}{F_{2}}},
$$

where $F_{\text {tot }}$ is the total flux of both stars and $F_{2}$ is the flux from the companion star. In Southworth \& Evans (2016), the error of the photometric light curve dominated the error calculation of their corrected transit depth and therefore we also use our photometric error bars for the error in the $\left(R_{p} / R_{*}\right)_{\text {cor }}$. Using this procedure we find an $\left(R_{p} / R_{*}\right)_{\text {cor }}=0.1181 \pm 0.0016$.

\subsection{XO-3b}

$\mathrm{XO}-3 \mathrm{~b}$ is a massive planet $\left(11.79 \pm 0.59 M_{\mathrm{Jup}}\right)$ with a large eccentricity $(0.26 \pm 0.017)$ detected by the XO survey (Johns-Krull et al. 2008). Further photometric observations have refined the system's parameters (Winn et al. 2008; Machalek et al. 2010; Hirano et al. 2011; Wong et al. 2014) and Hébrard et al. (2008) found that $\mathrm{XO}-3$ 's spin axis is misaligned with $\mathrm{XO}-3 \mathrm{~b}$ 's rotation axis.

We observed a transit of XO-3b on 2012 November 30 with the Harris $B$ filter (Fig. 3). We derive physical parameters that are in agreement with previous literature (Tables 5 and 7). Our calculated $R_{p} / R_{*}$ is $2 \sigma$ larger than the $V$-band $R_{p} / R_{*}$ found by Winn et al. (2008). We calculate a refined period with an error decreased by a factor of 13 from the value found by Winn et al. (2008). A non-flat spectrum for $R_{p} / R_{*}$ is found for $\mathrm{XO}-3 \mathrm{~b}$ (Table 9; Fig. 7).

\section{DISCUSSION}

\subsection{Wavelength dependence on the transit depth}

We find a constant transit depth across optical wavelengths for the TEPs HAT-P-5b, HAT-P-12b, WASP-2b, WASP-24b and WASP$80 \mathrm{~b}$ (Fig. 6, Table 9). A lack of variation in radius with wavelength could suggest these planets (HAT-P-5b, HAT-P-12b, WASP2b, WASP-80b) have clouds/hazes in their upper atmospheres (e.g. Seager \& Sasselov 2000; Brown 2001; Gibson et al. 2013b; Marley et al. 2013; Kreidberg et al. 2014) or they have an isothermal pressure-temperature profile (Fortney et al. 2006). Mancini et al. (2014) also do not detect a significant variation in WASP-80b's transit depth with wavelength, and Southworth et al. (2012) finds a relatively flat spectrum of planetary radii for HAT-P-5b with the exception of their observed radius in the $U$ band (which they suspect is caused by systematic error in their $U$-band photometry). A flat spectrum for WASP-24b is also found with the exception of one value. Our $R$-band $R_{p} / R_{*}$ found for WASP-24b differs by $4 \sigma$ from the previously calculated $R_{p} / R_{*}$ (Southworth et al. 2014) for that same band. The cause of this is unclear and future observations are needed to investigate. Our results are consistent with other transiting exoplanet observations having a flat spectrum in optical wavelengths (i.e. TrES-3b, Turner et al. 2013; GJ 1214b, Bean et al. 2011; Kreidberg et al. 2014; WASP-29b, Gibson et al. 2013a; Gibson et al. 2013b; HAT-P-19b, Mallonn et al. 2015a; HAT-P-1b, HAT-P-13b, HAT-P-16b, HAT-P-22b, TrES-2b, WASP-33b, WASP44b, WASP-48b, WASP-77Ab, Turner et al. 2016b).

We find variations in the transit depth with wavelength for CoRoT-12b, HAT-P-33b, HAT-P-37b, WASP-103b and XO-3b (Figs 6 and 7, Table 9), which could indicate scattering (i.e. due to aerosols or Rayleigh scattering) or absorption in their atmospheres (e.g. Benneke \& Seager 2012; Griffith 2014). Our observation of HAT-P-37b exhibits a smaller transit depth in $B$ band than the $\mathrm{red} /$ near-IR value. Such a variation has only been seen in a recent paper by Evans et al. (2016) where they observe a smaller $B$-band transit depth than optical in WASP-121b. Evans et al. (2016) believe a possible cause of such a variation is $\mathrm{TiO} / \mathrm{VO}$ absorption and this may also be the cause of the transit depth variations seen in HATP-37b. However, more theoretic modelling is needed to confirm 
Radius vs. Wavelength for HAT-P-5b
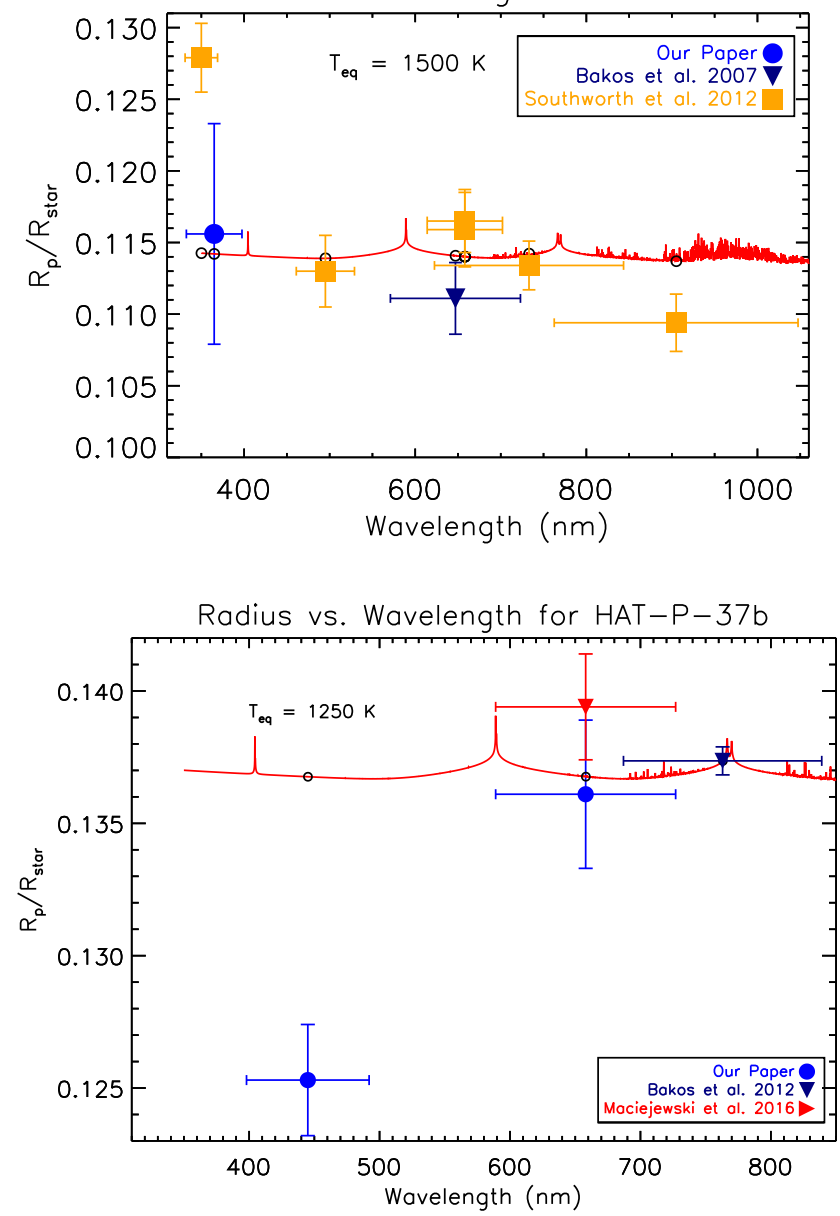

Radius vs. Wavelength for WASP $-24 b$

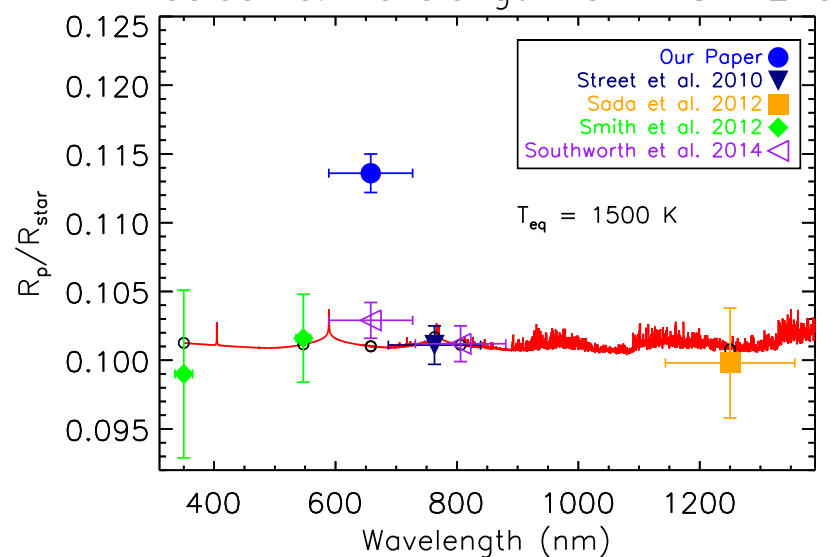

Radius vs. Wavelength for $H A T-P-12 b$

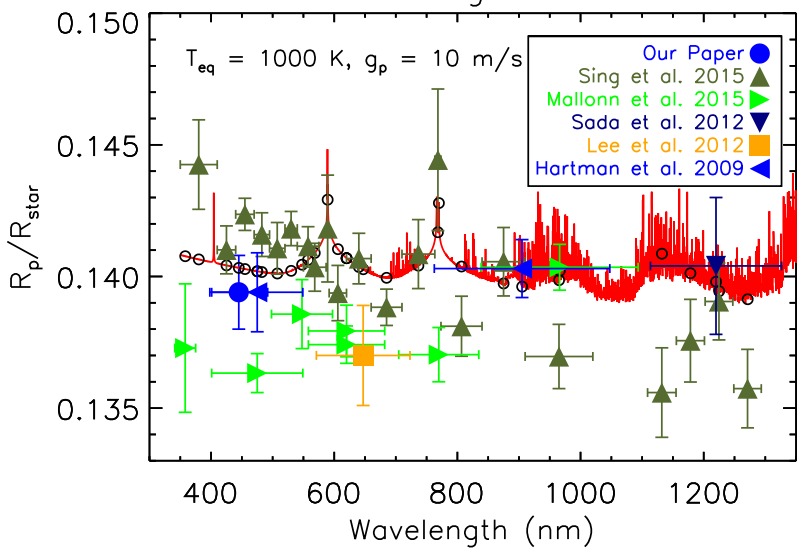

Radius vs. Wavelength for WASP-2b

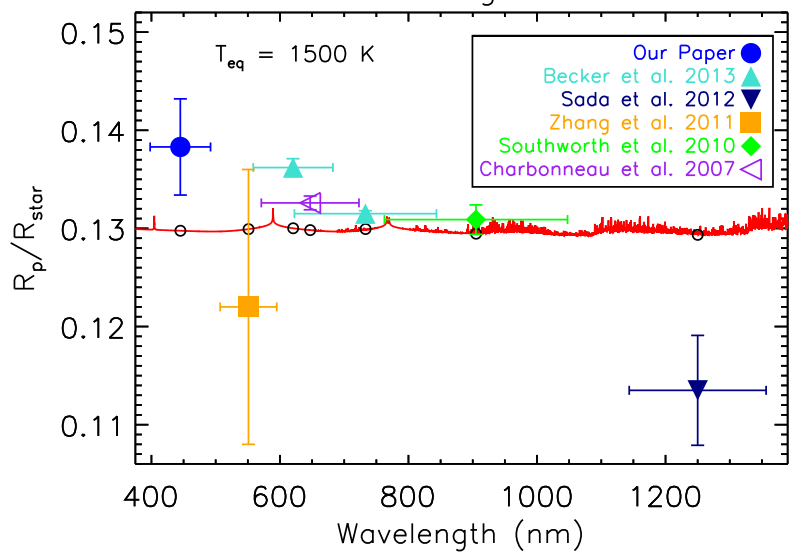

Radius vs. Wavelength for WASP-80b

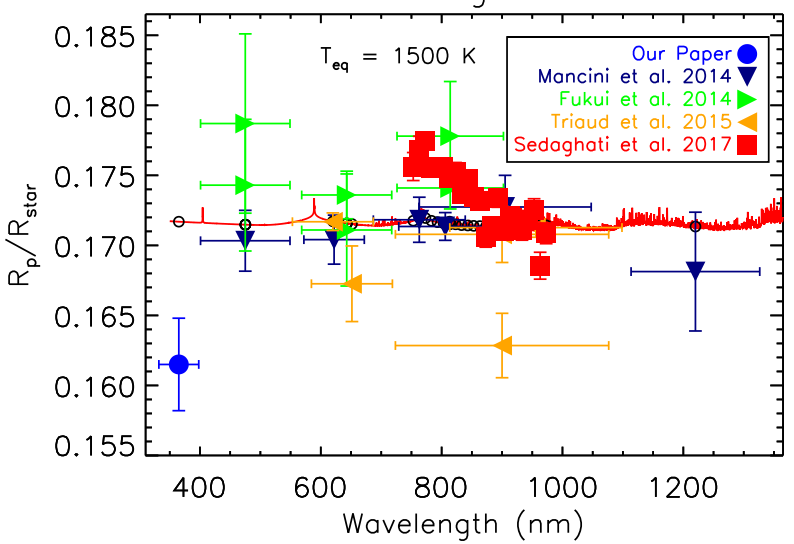

Figure 6. Plot of $R_{p} / R_{*}$ against wavelength for HAT-P-5b, HAT-P-12b, HAT-P-37b, WASP-2b, WASP-24b and WASP-80b from this paper and previous literature. Our data are shown as blue circles. Overplotted in red are atmospheric models by Fortney \& Nettelmann (2010) for planets with a $1 M_{\text {Jup }}, g_{p}=25$ $\mathrm{ms}^{-1}$ (unless specified on plot), $T_{\mathrm{eq}}$ (specified on plot) and a base radius of $1.25 R_{\mathrm{Jup}}$ at $10 \mathrm{bar}$. We find that HAT-P-5b, HAT-P-12b, WASP-2b and WASP-80b have flat spectra that could indicate the presence of clouds. The transit depth variation of HAT-P-37b could be due to absorption of TiO/VO (Evans et al. 2016).

that $\mathrm{TiO} / \mathrm{VO}$ is in fact the opacity source. Additionally, a smaller near-UV radius was recently observed in the hot Jupiter WASP-1b (Turner et al. 2016b); however, these observations did not observe in the $B$ band. Future near-UV and blue-band observations are needed for WASP-103b and XO-3b to determine whether the scattering in their atmospheres is due to Rayleigh scattering (Lecavelier Des Etangs et al. 2008; Tinetti et al. 2010; de Wit \& Seager 2013; Griffith 2014) since these bands are the only optical wavelengths that are not affected by strong spectral features. The radius variations in WASP-103b show a consistently larger transit depth in the 

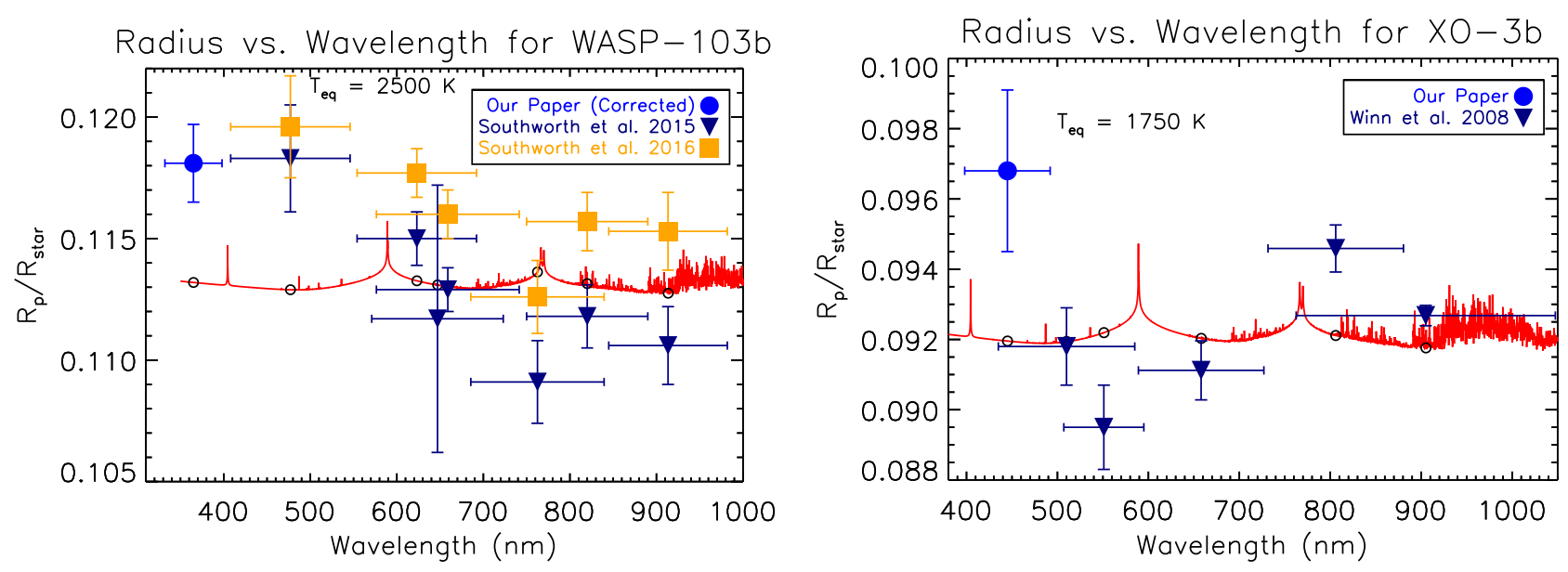

Figure 7. Plot of $\mathrm{R}_{p} / R_{*}$ against wavelength for WASP-103b and XO- $3 \mathrm{~b}$ from this paper and previous literature. Both WASP-103b and XO- $3 \mathrm{~b}$ show variations with wavelength. Other comments are the same as Fig. 6.

near-UV and blue than the rest of the optical (this variation is still present when corrected for dilution due the companion star). Such a radius variation may indicate a change in particle size at different altitudes of the planetary atmosphere (e.g. Wakeford \& Sing 2015). We find a larger $R$-band transit depth in HAT-P-33b and CoRoT-12b than their discovery transit depths. Since the $R$-filter encompasses the $\mathrm{H} \alpha$ line $(656.281 \mathrm{~nm})$, our observation could be an indication of atmospheric escape such as that observed in the atmospheres of HD 189733b (Jensen et al. 2012; Cauley et al. 2015; Barnes et al. 2016; Cauley, Redfield \& Jensen 2017a; Cauley, Redfield \& Jensen 2017b) and HD 209458b (Astudillo-Defru \& Rojo 2013) and predicted (e.g. Christie, Arras \& Li 2013; Turner et al. 2016a). Follow-up photometry and high-resolution spectroscopy observations are encouraged to confirm all the transit depth variations. These results also agree with observations of other exoplanets not having a flat spectrum (i.e. HD 209458b, Sing et al. 2008; HATP-5b, Southworth et al. 2012; GJ 3470b, Nascimbeni et al. 2013; Qatar-2, Mancini et al. 2014; WASP-17b, WASP-39b, HAT-P-1b, WASP-31b, HAT-P-12b, HD189733b, WASP-6b, Sing et al. 2016; CoRoT-1b, TrES-4b, WASP-1b, WASP-12b, WASP-36b, Turner et al. 2016b).

For illustration, the observed $R_{p} / R_{*}$ differences with wavelength for each target (Table 9) are compared to theoretical predictions (Fortney \& Nettelmann 2010) for a model planetary atmosphere (Figs 6 and 7). The models used are calculated for planets with a $1 M_{\text {Jup }}, g_{p}=25 \mathrm{~ms}^{-1}$ or $g_{p}=10 \mathrm{~ms}^{-1}$, base radius of $1.25 R_{\text {Jup }}$ at $10 \mathrm{bar}, T_{\mathrm{eq}}$ closest to the measured value for each exoplanet (with model choices of 500, 750, 1000, 1250, 1500, 1750, 2000, $2500 \mathrm{~K}$ ), and solar metallicity. To provide a best fit to the spectral changes, a vertical offset is applied to the model. This comparison is helpful as it illustrates the size of observed variation compared to what the theoretical models predict. However, radiative transfer models calculated for each exoplanet individually are needed to fully understand their transmission spectra.

Finally, no signs of asymmetric transits are seen in the nearUV light curves of HAT-P-5b, WASP-80b and WASP-103b. This result is consistent with ground-based near-UV observations of 19 other transiting exoplanets (Southworth et al. 2012; Copperwheat et al. 2013; Turner et al. 2013; Bento et al. 2014; Pearson et al. 2014; Zellem et al. 2015; Turner et al. 2016b) which show no evidence of asymmetric transits. Additionally, theoretical modelling by Turner et al. (2016a) using the CLOUDY plasma simulation code showed that asymmetric transits cannot be produced in the broad-band nearUV band regardless of the assumed physical phenomena that could cause absorption (e.g. Lai, Helling \& van den Heuvel 2010; Vidotto, Jardine \& Helling 2010; Ben-Jaffel \& Ballester 2014; Matsakos, Uribe \& Königl 2015; Kislyakova et al. 2016).

\subsubsection{Variability in the host stars}

One of the major assumptions in our interpretation that the planetary atmosphere is the cause of the transit depth variations is that the brightness of the host stars have minimal variability due to stellar activity. The presence of star spots and stellar activity can produce variations in the observed transit depth (e.g. Czesla et al. 2009; Oshagh et al. 2013, 2014; Zellem et al. 2015, 2017). This effect is stronger in the near-UV and blue and can mimic a Rayleigh scattering signature (e.g. Oshagh et al. 2014; McCullough et al. 2014). Additionally, no obvious star spot crossing is seen in our data (Figs 1-3) with the possible exception of HAT-P-37b (see below).

We estimate how much the transit depth may change due to unocculted spots using the formalization presented by Sing et al. (2011). This method assumes that the spots can be treated as a stellar spectrum but with a lower effective temperature, no surface brightness variation outside the spots and no plage are present. The effect of these assumptions are a dimming of the star and therefore an increase in the transit depth. Sing et al. (2011) find for HD $189733 \mathrm{~b}$ that the change in transit depth due to unocculted spots, $\Delta\left(R_{p} / R_{*}\right)=2.08 \times 10^{-3} / 2\left(R_{p} / R_{*}\right)$ between 375 and 400 $\mathrm{nm}$. Therefore, unocculted spots have minimal influence (assuming our host stars have unocculted spots similar to HD 189733b) on the observed transit depth variations since our final error bars (Table 5) are at least 10 times larger than the influence of these spots (e.g. $\Delta\left[R_{p} / R_{*}\right]=0.00014$ for HAT-P-37b). Qualitatively, this result is consistent with the study by Llama \& Shkolnik (2015) that find that stellar activity similar to the sun has very little effect on the transit depth measured in near-UV to optical wavelengths. None the less, we highly encourage follow-up observations and host star monitoring of all our targets to assess the effect of stellar activity on the observed transit depth variations.

Next, we investigate what effect a star-spot crossing in the light curve of HAT-P-37 would have on its calculated transit depth. In the $B$-band light curve of HAT-P-37b (Fig. 1), there may be a star-spot 
crossing at a phase range of $0.004-0.008$. However, the detected signal is very close to the scatter in the light curve. If we model the light curve without the possible star-spot crossing, we find an $\left(R_{p} / R_{*}\right)=0.1278 \pm 0.0048$, within $1 \sigma$ of the transit depth of the entire light curve $(0.1253 \pm 0.0021)$. McCullough et al. (2014) present a procedure to estimate the effects of unocculted spots on the transit depth. Their procedure can also be used to estimate the effect of star spot crossings on the transit depth, where instead of unocculted spots increasing the transit depth occulted spots should decrease it. McCullough et al. (2014) find that the change in transit depth due to spots, $\Delta\left(R_{p}^{2} / R_{*}^{2}\right)$, is

$\Delta\left(\frac{R_{p}^{2}}{R_{*}^{2}}\right)=\left(\frac{R_{p}}{R_{*}}\right)^{2} \delta \frac{T_{\text {spot }}}{T_{\text {eff }}}$,

where $R_{p} / R_{*}$ is the unperturbed transit depth, $\delta$ is the fractional area of star spots and $T_{\text {spot }}$ is the temperature of the spot. If we set $\Delta\left(R_{p}^{2} / R_{*}^{2}\right)=3100 \mathrm{ppm}$ (the approximate difference between our $B$-band and the Sloan- $i$ transit depth; Hartman et al. 2011), then we can estimate $T_{\text {spot }}$ and $\delta$. For spot temperatures between 2000 and $5000 \mathrm{~K}$, we find that $\delta$ would be between 20 and 50 percent. Typical values of $\delta$ for solar-like stars is around several percent (e.g. Pont et al. 2008; Sing et al. 2011; McCullough et al. 2014), so our estimated $\delta$ range is extremely high. Due to both these tests, it seems unlikely that the smaller $B$-band transit depth of HAT-P-37b is due to an occulted star-spot.

\section{CONCLUSIONS}

We observed 11 transiting hot Jupiters (CoRoT-12b, HAT-P5b, HAT-P-12b, HAT-P-33b, HAT-P-37b, WASP-2b, WASP-24b, WASP-60b, WASP-80b, WASP-103b, XO-3b) from the ground using near-UV and optical filters in order to update their system parameters and constrain their atmospheres. Our observations of CoRoT-12b, HAT-P-37b and WASP-60b are the first follow-up observations of these planets since their discovery and we also obtain the first near-UV light curves of WASP-80b and WASP-103b. We find that HAT-P-5b, HAT-P-12b, WASP-2b, WASP-24b and WASP$80 \mathrm{~b}$ exhibit a flat spectrum across the optical wavelengths, suggestive of clouds in their atmospheres. Variation in the transit depths is observed for WASP-103b and XO-3b and may indicate scattering in their atmospheres. Additionally, we observe a smaller $B$-band transit depth compared to near-IR in HAT-P-37b. Such a variation may be caused by TiO/VO absorption (Evans et al. 2016). We find a larger $R$-band (which encompasses the $\mathrm{H} \alpha$ line) transit depths in HAT-P-33b and CoRoT-12b and this result may indicate possible atmospheric escape. Follow-up photometry and high-resolution spectroscopy observations are encouraged to confirm all the observed transit depth variations since they are only seen at $2 \sigma-4.6 \sigma$. Our calculated physical parameters agree with previous studies within $1 \sigma$ with a few exceptions (Tables 6 and 7). For the exoplanets HATP-12b, HAT-P-37b, WASP-2b, WASP-24b, WASP-80b and XO$3 \mathrm{~b}$, we are able to refine their orbital periods from previous work (Tables 6 and 7).

\section{ACKNOWLEDGEMENTS}

JT, RL and RJ were partially supported by the NASA's Planetary Atmospheres programme. JT and RL were also supported by The Double Hoo Research Grant. JT was also partially funded by the National Science Foundation Graduate Research Fellowship under grant no. DGE-1315231.
We would like thank Robert T. Zellem for his help with observations. This research has made use of the Exoplanet Orbit Database (Wright et al. 2011), Exoplanet Data Explorer at exoplanets.org, Exoplanet Transit Database, Extrasolar Planet Transit Finder, NASA's Astrophysics Data System Bibliographic Services and the International Variable Star Index (VSX) data base, operated at AAVSO, Cambridge, Massachusetts, USA. This research has also made use of the NASA Exoplanet Archive, which is operated by the California Institute of Technology, under contract with the National Aeronautics and Space Administration under the Exoplanet Exploration Program. We also thank the anonymous referee, whose comments helped improve this paper.

\section{REFERENCES}

Akeson R. L. et al., 2013, PASP, 125, 989

Albrecht S. et al., 2011, ApJ, 738, 50

Astudillo-Defru N., Rojo P., 2013, A\&A, 557, A56

Baglin A., 2003, Adv. Space Res., 31, 345

Bakos G. Á. et al., 2007, ApJ, 671, L173

Bakos G. Á. et al., 2012, AJ, 144, 19

Barnes J. W., van Eyken J. C., Jackson B. K., Ciardi D. R., Fortney J. J., 2013, ApJ, 774, 53

Barnes J. R., Haswell C. A., Staab D., Anglada-Escudé G., 2016, MNRAS, 462,1012

Bean J. L. et al., 2011, ApJ, 743, 92

Becker A. C., Kundurthy P., Agol E., Barnes R., Williams B. F., Rose A. E., 2013, ApJ, 764, L17

Ben-Jaffel L., Ballester G. E., 2014, ApJ, 785, L30

Benneke B., Seager S., 2012, ApJ, 753, 100

Bento J. et al., 2014, MNRAS, 437, 1511

Bessell M. S., 1979, PASP, 91, 589

Biddle L. I. et al., 2014, MNRAS, 443, 1810

Borucki W. J. et al., 2010, Science, 327, 977

Braak C., 2006, Stat. Comput., 16, 239

Brown T. M., 2001, ApJ, 553, 1006

Bruntt H., Southworth J., Torres G., Penny A. J., Clausen J. V., Buzasi D. L., 2006, A\&A, 456, 651

Carone L. et al., 2012, A\&A, 538, A112

Carter J. A., Winn J. N., 2009, ApJ, 704, 51

Castelli F., Kurucz R. L., 2004, preprint (astro-ph/0405087)

Cauley P. W., Redfield S., Jensen A. G., Barman T., Endl M., Cochran W. D., 2015, ApJ, 810, 13

Cauley P. W., Redfield S., Jensen A. G., 2017a, AJ, 153, 185

Cauley P. W., Redfield S., Jensen A. G., 2017b, AJ, 153, 217

Charbonneau D., Brown T. M., Latham D. W., Mayor M., 2000, ApJ, 529, L45

Charbonneau D., Brown T. M., Noyes R. W., Gilliland R. L., 2002, ApJ, 568,377

Charbonneau D., Brown T. M., Burrows A., Laughlin G., 2007, Protostars and Planets V. Univ. Arizona Press, Tucson, AZ, p. 701

Christie D., Arras P., Li Z.-Y., 2013, ApJ, 772, 144

Ciardi D. R., Beichman C. A., Horch E. P., Howell S. B., 2015, ApJ, 805, 16

Claret A., Bloemen S., 2011, A\&A, 529, A75

Collier Cameron A. et al., 2007, MNRAS, 375, 951

Copperwheat C. M. et al., 2013, MNRAS, 434, 661

Czesla S., Huber K. F., Wolter U., Schröter S., Schmitt J. H. M. M., 2009 , A\&A, 505, 1277

Daemgen S., Hormuth F., Brandner W., Bergfors C., Janson M., Hippler S., Henning T., 2009, A\&A, 498, 567

de Wit J., Seager S., 2013, Science, 342, 1473

Dittmann J. A., Close L. M., Green E. M., Scuderi L. J., Males J. R., 2009a, ApJ, 699, L48

Dittmann J. A., Close L. M., Green E. M., Fenwick M., 2009b, ApJ, 701, 756 
Dittmann J. A., Close L. M., Scuderi L. J., Morris M. D., 2010, ApJ, 717, 235

Dittmann J. A., Close L. M., Scuderi L. J., Turner J., Stephenson P. C., 2012, New Astron., 17, 438

Eastman J., Siverd R., Gaudi B. S., 2010, PASP, 122, 935

Eastman J., Gaudi B. S., Agol E., 2013, PASP, 125, 83

Evans T. M. et al., 2016, ApJ, 822, L4

Ford E. B., 2006, ApJ, 642, 505

Fortney J. J., Nettelmann N., 2010, Space Sci. Rev., 152, 423

Fortney J. J., Cooper C. S., Showman A. P., Marley M. S., Freedman R. S., 2006, ApJ, 652, 746

Fortney J. J., Marley M. S., Barnes J. W., 2007, ApJ, 659, 1661

Fukui A. et al., 2014, ApJ, 790, 108

Gelman A., Rubin D. B., 1992, Stat. Sci., 7, 457

Gibson N. P., Aigrain S., Barstow J. K., Evans T. M., Fletcher L. N., Irwin P. G. J., 2013a, MNRAS, 428, 3680

Gibson N. P., Aigrain S., Barstow J. K., Evans T. M., Fletcher L. N., Irwin P. G. J., 2013b, MNRAS, 436, 2974

Gillon M. et al., 2010, A\&A, 520, A97

Gillon M. et al., 2014, A\&A, 562, L3

Griffith C. A., 2014, Phil. Trans. R. Soc. A, 372, 30086

Hartman J. D. et al., 2009, ApJ, 706, 785

Hartman J. D. et al., 2011, ApJ, 742, 59

Hébrard G. et al., 2008, A\&A, 488, 763

Hébrard G. et al., 2013, A\&A, 549, A134

Henry G. W., Marcy G. W., Butler R. P., Vogt S. S., 2000, ApJ, 529, L41

Hirano T., Narita N., Sato B., Winn J. N., Aoki W., Tamura M., Taruya A., Suto Y., 2011, PASJ, 63, L57

Holman M. J., Murray N. W., 2005, Science, 307, 1288

Holman M. J. et al., 2010, Science, 330, 51

Howell S. B. et al., 2014, PASP, 126, 398

Hubbard W. B., Fortney J. J., Lunine J. I., Burrows A., Sudarsky D., Pinto P., 2001, ApJ, 560, 413

Husnoo N., Pont F., Mazeh T., Fabrycky D., Hébrard G., Bouchy F., Shporer A., 2012, MNRAS, 422, 3151

Jensen A. G., Redfield S., Endl M., Cochran W. D., Koesterke L., Barman T., 2012, ApJ, 751, 86

Johns-Krull C. M. et al., 2008, ApJ, 677, 657

Kislyakova K. G. et al., 2016, MNRAS, 461, 988

Kreidberg L. et al., 2014, Nature, 505, 69

Lai D., Helling C., van den Heuvel E. P. J., 2010, ApJ, 721, 923

Lecavelier Des Etangs A., Pont F., Vidal-Madjar A., Sing D., 2008, A\&A, 481, L83

Lee J. W., Youn J.-H., Kim S.-L., Lee C.-U., Hinse T. C., 2012, AJ, 143, 95

Lendl M., Cubillos P. E., Hagelberg J., Müller A., Juvan I., Fossati L., 2017, preprint (arXiv:1708.05737)

Line M. R., Knutson H., Deming D., Wilkins A., Desert J.-M., 2013, ApJ, 778,183

Llama J., Shkolnik E. L., 2015, ApJ, 802, 41

McCullough P. R., Crouzet N., Deming D., Madhusudhan N., 2014, ApJ, 791, 55

Machalek P., Greene T., McCullough P. R., Burrows A., Burke C. J., Hora J. L., Johns-Krull C. M., Deming D. L., 2010, ApJ, 711, 111

Maciejewski G. et al., 2016, Acta Astron., 66, 55

Mallonn M. et al., 2015a, A\&A, 580, A60

Mallonn M. et al., 2015b, A\&A, 583, A138

Mancini L. et al., 2014, A\&A, 562, A126

Mandel K., Agol E., 2002, ApJ, 580, L171

Markwardt C. B., 2009, in Bohlender D. A., Durand D., Dowler P., eds, ASP Conf. Ser. Vol. 411, Astronomical Data Analysis Software and Systems XVIII. Astron. Soc. Pac., San Francisco, p. 251

Marley M. S., Ackerman A. S., Cuzzi J. N., Kitzmann D., 2013, Clouds and Hazes in Exoplanet Atmospheres. Univ. Arizona Press, Tucson, AZ, p. 367

Matsakos T., Uribe A., Königl A., 2015, A\&A, 578, A6

Miralda-Escudé J., 2002, ApJ, 564, 1019

Moutou C. et al., 2013, Icarus, 226, 1625
Nascimbeni V., Piotto G., Pagano I., Scandariato G., Sani E., Fumana M., 2013, A\&A, 559, A32

Ngo H. et al., 2016, ApJ, 827, 8

Oshagh M., Santos N. C., Boisse I., Boué G., Montalto M., Dumusque X., Haghighipour N., 2013, A\&A, 556, A19

Oshagh M., Santos N. C., Ehrenreich D., Haghighipour N., Figueira P., Santerne A., Montalto M., 2014, A\&A, 568, A99

Parviainen H. et al., 2017, preprint (arXiv:1709.01875)

Pearson K. A., Turner J. D., Sagan T. G., 2014, New Astron., 27, 102

Pollacco D. L. et al., 2006, PASP, 118, 1407

Pont F., Zucker S., Queloz D., 2006, MNRAS, 373, 231

Pont F., Knutson H., Gilliland R. L., Moutou C., Charbonneau D., 2008, MNRAS, 385, 109

Press W. H., Teukolsky S. A., Vetterling W. T., Flannery B. P., 1992, Numerical Recipes in FORTRAN. The Art of Scientific Computing. Cambridge Univ. Press, Cambridge

Saar S. H., Butler R. P., Marcy G. W., 1998, ApJ, 498, L153

Sada P. V., Ramón-Fox F. G., 2016, PASP, 128, 024402

Sada P. V. et al., 2012, PASP, 124, 212

Safronov V. S., 1972, Evolution of the Protoplanetary Cloud and Formation of the Earth and Planets. Keter Publ. House, Jerusalem, p. 212

Salz M., Schneider P. C., Czesla S., Schmitt J. H. M. M., 2015, A\&A, 576, A42

Schwarz G., 1978, Ann. Stat., 6, 461

Scuderi L. J., Dittmann J. A., Males J. R., Green E. M., Close L. M., 2010, ApJ, 714, 462

Seager S., 2011, Exoplanets. Univ. Arizona Press, Tucson, AZ

Seager S., Sasselov D. D., 2000, ApJ, 537, 916

Sedaghati E., Boffin H. M. J., Delrez L., Gillon M., Csizmadia S., Smith A. M. S., Rauer H., 2017, MNRAS, 468, 3123

Simpson E. K. et al., 2011, MNRAS, 414, 3023

Sing D. K., Vidal-Madjar A., Désert J.-M., Lecavelier des Etangs A., Ballester G., 2008, ApJ, 686, 658

Sing D. K. et al., 2011, MNRAS, 416, 1443

Sing D. K. et al., 2016, Nature, 529, 59

Sokov E. N. et al., 2012, Astron. Lett., 38, 180

Southworth J., 2008, MNRAS, 386, 1644

Southworth J., 2010, MNRAS, 408, 1689

Southworth J., Evans D. F., 2016, MNRAS, 463, 37

Southworth J., Wheatley P. J., Sams G., 2007a, MNRAS, 379, L11

Southworth J., Bruntt H., Buzasi D. L., 2007b, A\&A, 467, 1215

Southworth J. et al., 2010, MNRAS, 408, 1680

Southworth J., Mancini L., Maxted P. F. L., Bruni I., Tregloan-Reed J., Barbieri M., Ruocco N., Wheatley P. J., 2012, MNRAS, 422, 3099

Southworth J. et al., 2014, MNRAS, 444, 776

Southworth J. et al., 2015, MNRAS, 447, 711

Street R. A. et al., 2010, ApJ, 720, 337

Teske J. K., Turner J. D., Mueller M., Griffith C. A., 2013, MNRAS, 431, 1669

Tinetti G., Deroo P., Swain M. R., Griffith C. A., Vasisht G., Brown L. R., Burke C., McCullough P., 2010, ApJ, 712, L139

Torres G., Winn J. N., Holman M. J., 2008, ApJ, 677, 1324

Triaud A. H. M. J. et al., 2010, A\&A, 524, A25

Triaud A. H. M. J. et al., 2013, A\&A, 551, A80

Triaud A. H. M. J. et al., 2015, MNRAS, 450, 2279

Turner J. D. et al., 2013, MNRAS, 428, 678

Turner J. D., Christie D., Arras P., Johnson R. E., Schmidt C., 2016a, MNRAS, 458, 3880

Turner J. D. et al., 2016b, MNRAS, 459, 789

Vidotto A. A., Jardine M., Helling C., 2010, ApJ, 722, L168

Wakeford H. R., Sing D. K., 2015, A\&A, 573, A122

Wang Y.-H. et al., 2017, AJ, 154, 49

Winn J. N., 2011, in Seager S., ed., Exoplanet Transits and Occultations. Univ. Arizona Press, Tucson, AZ

Winn J. N. et al., 2008, ApJ, 683, 1076

Wöllert M., Brandner W., 2015, A\&A, 579, A129

Wong I. et al., 2014, ApJ, 794, 134 
Wright J. T. et al., 2011, PASP, 123, 412

Zellem R. T. et al., 2015, ApJ, 810, 11

Zellem R. T. et al., 2017, ApJ, 844, 27

Zhang J.-C., Cao C., Song N., Wang F.-G., Zhang X.-T., 2011, Chin. Astron. Astrophys., 35, 409

\section{SUPPORTING INFORMATION}

Supplementary data are available at MNRAS online.

Table 2. Photometry of all our light curves.

Table 8. Results of the transit timing analysis.
Table 9. $R_{p} / R_{*}$ and effective central wavelength $\lambda_{\text {eff }}$ from this paper and previous literature for all targets.

Please note: Oxford University Press is not responsible for the content or functionality of any supporting materials supplied by the authors. Any queries (other than missing material) should be directed to the corresponding author for the article.

This paper has been typeset from a $\mathrm{T}_{\mathrm{E}} \mathrm{X} / \mathrm{L} \mathrm{T}_{\mathrm{E}} \mathrm{X}$ file prepared by the author. 